ESAIM: M2AN 46 (2012) 1003-1028

DOI: $10.1051 / \mathrm{m} 2 \mathrm{an} / 2011046$
ESAIM: Mathematical Modelling and Numerical Analysis

www.esaim-m2an.org

\title{
A FAMILY OF DISCONTINUOUS GALERKIN MIXED METHODS FOR NEARLY AND PERFECTLY INCOMPRESSIBLE ELASTICITY*
}

\author{
YongXing Shen ${ }^{1}$ AND AdRian J. Lew ${ }^{2}$
}

\begin{abstract}
We introduce a family of mixed discontinuous Galerkin (DG) finite element methods for nearly and perfectly incompressible linear elasticity. These mixed methods allow the choice of polynomials of any order $k \geq 1$ for the approximation of the displacement field, and of order $k$ or $k-1$ for the pressure space, and are stable for any positive value of the stabilization parameter. We prove the optimal convergence of the displacement and stress fields in both cases, with error estimates that are independent of the value of the Poisson's ratio. These estimates demonstrate that these methods are locking-free. To this end, we prove the corresponding inf-sup condition, which for the equal-order case, requires a construction to establish the surjectivity of the space of discrete divergences on the pressure space. In the particular case of near incompressibility and equal-order approximation of the displacement and pressure fields, the mixed method is equivalent to a displacement method proposed earlier by Lew et al. [Appel. Math. Res. express 3 (2004) 73-106]. The absence of locking of this displacement method then follows directly from that of the mixed method, including the uniform error estimate for the stress with respect to the Poisson's ratio. We showcase the performance of these methods through numerical examples, which show that locking may appear if Dirichlet boundary conditions are imposed strongly rather than weakly, as we do here.
\end{abstract}

Mathematics Subject Classification. 65N30, 65N12, 65N15.

Received January 22, 2011. Revised June 12, 2011.

Published online February 13, 2012.

\section{INTRODUCTION}

Volumetric locking limits the use of the otherwise robust standard conforming finite element method (FEM) for solving elasticity boundary value problems involving an incompressible or nearly incompressible elasticity tensor. The underlying reason for this phenomenon is the inability of the divergence-free subspace of low-order (piecewise cubic or lower) conforming approximation spaces to approxiate divergence-free displacement fields to the optimal order.

\footnotetext{
Keywords and phrases. Discontinuous Galerkin, locking, mixed method, inf-sup condition.

* Department of the Army Research Grant, grant number: W911NF-07-2-0027; NSF Career Award, grant number: CMMI-0747089; ONR Young Investigator Award, grant number: N000140810852.

1 Laboratori de Càlcul Numèric, Universitat Politècnica de Catalunya (UPC BarcelonaTech), Barcelona, Spain. yongxing. shen@upc.edu

2 Department of Mechanical Engineering, Stanford University, Stanford, 94305-4040 California, USA.

lewa@stanford.edu
} 
Precisely the same problem found in incompressible elasticity is found in Stokes flow. Both problems are described by precisely the same set of equations, but in the latter the velocity field plays the role of the elastic displacement field. Therefore, in the following we will refer to literature on methods for both problems.

Most methods that are able to overcome the locking difficulty can be categorized as either a displacement method or a mixed method, depending on whether the displacement field is the only unknown field to solve for with the primary equations or not. In order for a displacement method to have a uniform error estimate in the incompressible limit $\lambda \rightarrow \infty$ where $\lambda$ is the Lamé parameter, a conforming $\left(C^{0}\right)$ method requires that the approximation space contain at least piecewise quartic polynomials in the approximation space [34]; a non-conforming method does not have such a restrictive requirement, and hence is more attractive. Early nonconforming approaches in two-dimensions include the displacement methods developed by Brenner and Sung [9] and Kouhia and Stenberg [29]. These methods were based on the piecewise affine Crouzeix-Raviart element [20], which enforces inter-element continuity of the displacement only at edge midpoints.

An important family of non-conforming FEMs is that of discontinuous Galerkin (DG) methods, which assume no continuity between neighboring elements. These have also been used as bases for building locking-free methods with displacement formulations, such as the two Nitsche-type DG methods developed by Hansbo and Larson $[23,24]$ and the non-symmetric interior penalty DG method proposed by Wihler [40]. In these contributions, both the absence of locking and the optimal convergence in displacement were proved by virtue of a divergence-free interpolant, such as the Brezzi-Douglas-Marini (BDM) interpolant (for [23]) and the CrouzeixRaviart interpolant (for $[24,40]$ ).

Displacement methods are generally difficult to formulate for the perfectly incompressible case. While near incompressibility requires the approximation space of a displacement method to contain a divergence-free subspace, perfect incompressibility usually demands the entire basis to be divergence free (see, e.g., $[26,38]$ ), or at least elementwise divergence free (see, for instance, the DG methods developed by Hansbo and Larson [25]).

Another category of methods, the mixed methods, essentially imposes the (nearly) incompressible condition weakly. In general, while the displacement solution obtained from mixed methods is usually of good quality, the pressure solution may suffer from the checkerboard oscillation or other instability problems, unless an inf-sup condition is satisfied [10]. Proving this condition for a particular combination of displacement and pressure spaces is a delicate task, especially for conforming spaces. Notable stable combinations of displacement-pressure spaces (or velocity-pressure spaces in the context of Stokes flow) include the Taylor-Hood element [27,28] (first analyzed by Bercovier and Pironneau [5]), the Crouzeix-Raviart element [20], and the MINI element [1].

Recent mixed methods with a DG space for the displacement (or equivalently, the velocity in the Stokes equation) include the local DG (LDG) methods by Cockburn et al. $[18,19]$, the hybridized globally divergencefree LDG methods by Carrero et al. [16], the hybridizable DG methods by Soon et al. [35] and Nguyen et al. [31], the DG methods with non-overlapping subdomains by Girault et al. [22] and Rivière and Girault [32], and the mixed method proposed by Becker et al. [4] for problems involving discontinuous elastic moduli with at least one incompressible material. The reader is also referred to an abstract framework for a few mixed DG methods for the Stokes problem by Schötzau et al. [33].

In this paper, we propose a family of DG mixed methods in a unified framework for both near and perfect incompressibility. It accommodates equal order approximations for displacement and pressure fields, as well as the adoption of pressure approximations that are one polynomial degree smaller than those for the displacement. We prove the optimal convergence properties of the proposed mixed methods with error bound for displacement and stresses that are uniform for all values of $\lambda$ in $[\mu, \infty]$, where $\mu>0$ is the shear modulus of the material. Additionally, we compare the performance of these methods through numerical examples, including an investigation of the effects of strong versus weak enforcement of Dirichlet boundary conditions. Perhaps surprisingly, the examples show that weakly imposed Dirichlet boundary conditions are essential to avoid locking.

In the case of equal order approximations and nearly incompressible materials, the method is essentially the same as the displacement method in [30], except for the form of the stabilization term. This last method was in turn based on the method for the scalar Laplacian in [14]. An important advantage of these mixed methods is 
the problem-independence in the choice of the stabilization parameter - the methods are stable for any positive value of the stabilization parameter.

One distinct feature of our methods is that we equip the displacement space with an operator $D_{D G}$, the $D G$ derivative operator, in order to formally differentiate functions in this space. This operator reduces to the classical gradient operator $\nabla$ for $C^{0}$ functions that satisfy the Dirichlet boundary conditions, and hence is a generalization of $\nabla$ for the DG displacement space. The main advantage offered by this operator in this case is a systematic way to approximate the stress by replacing $\nabla$ in the constitutive equation by $D_{D G}$ for the discontinuous displacement solution. The resulting stress solution converges optimally and the error bound is uniform with $\lambda$ for a given choice of the polynomial order. In contrast, the stress approximation obtained merely by differentiating the displacement solution within each element does not enjoy this property. For equal order approximations, the $D_{D G}$ operator coincides with the standard expression for the approximation of derivatives in DG methods through the use of lifting operators (see, e.g., [2]) and the so-called Bassi-Rebay numerical fluxes [3].

A generalization of the equal-order method here to nonlinear elasticity in the compressible range was introduced by Ten Eyck and Lew [36], and their numerical results for nearly incompressible materials did not show traces of the locking problem. A related method for incompressible nonlinear elastic materials was proposed by Whiteley [39]. No signs of locking were observed in the numerical examples therein either. The results of our analysis should serve as a step towards the analysis of these more complex scenarios.

As mentioned earlier, we allow a certain degree of flexibility to choose the order of polynomials to approximate the displacement and the pressure. Precisely, the order of the polynomial for the pressure can be either the same as, or one order lower than, that for the displacement. These choices are abbreviated here as $P_{k} / P_{k}$ and $P_{k} / P_{k-1}$, respectively, where $k \in \mathbb{N}$. These two combinations yield the same orders of convergence. Even though they do not yield higher order approximations, there are two reasons to analyze the $P_{k} / P_{k}$ combination, besides that of merely exploring the possibilities of stable displacement/pressure combinations. First, in the case of $\lambda=\infty$, the DG version of the incompressibility condition is strictly enforced when we choose $P_{k} / P_{k}$, i.e., $\operatorname{div}_{D G} \boldsymbol{u}_{h} \equiv \operatorname{tr} D_{D G} \boldsymbol{u}_{h}=0$, where $\boldsymbol{u}_{h}$ is the displacement solution. Second, as mentioned earlier, when $\lambda<\infty$, the $P_{k} / P_{k}$ setting is equivalent to the displacement method proposed in $[30,37]$; therefore, the result of the analysis of the mixed method also holds for this displacement method, including a $\lambda$-uniform error estimate for the stress, which significantly strengthens the result of the analysis in [30].

The critical step in the forthcoming analysis is the proof of the inf-sup condition. While the satisfaction of this condition with the combination $P_{k} / P_{k-1}$ is a direct consequence of the definition of the BDM element, the proof for the stronger result with $P_{k} / P_{k}$ needs some delicate constructions. The essential step of such constructions is the proof of the surjectivity of the DG divergence operator $\operatorname{div}_{D G}$ onto the pressure space.

A few more ingredients are needed in order to obtain the desired error estimates. One of such ingredients is that, given any displacement field $\boldsymbol{u}$ such that $\operatorname{div} \boldsymbol{u}=0$, there always exists $\boldsymbol{u}_{I}$, a member of the DG approximation space, such that: (a) $\operatorname{div}_{D G} \boldsymbol{u}-\operatorname{div}_{D G} \boldsymbol{u}_{I}=0$, and (b) $\boldsymbol{u}-\boldsymbol{u}_{I}$ approaches zero at an optimal rate with respect to $h$. This construction is given by the BDM interpolation. A second ingredient is that the consistency error which arises from the lack of Galerkin orthogonality of the method also approaches zero at the optimal rate. A third ingredient is the weak enforcement of the Dirichlet boundary conditions. We will show by a numerical example that strong enforcement (e.g., by nodal interpolation) of the Dirichlet boundary conditions may lead to locking.

The rest of the paper is organized as follows: in Section 2 we state the problems and introduce the regularity assumptions for our subsequent analysis; then in Section 3 we introduce our mixed method and recapitulate the related displacement method proposed in [30]; Section 4 is devoted to the proofs of convergence and absence of locking; finally in Section 5, we present the numerical examples and show the possible detrimental effect of strong enforcement of the Dirichlet boundary conditions. 


\section{Problem statement and Regularity assumptions}

\subsection{The linear elastostatic problem}

We consider a two- or three-dimensional linear isotropic elastostatic problem over a bounded convex open polyhedral domain $B \subset \mathbb{R}^{d}, d=2,3$, with a Lipschitz continuous boundary $\partial B$. The problem reads: find $(\boldsymbol{u}, p) \in\left[H^{1}(B)\right]^{d} \times L^{2}(B)$ such that

$$
\begin{aligned}
-\operatorname{div} \sigma & =\boldsymbol{f}, & \text { in } B, \\
\sigma & =2 \mu \nabla^{\mathrm{sym}} \boldsymbol{u}-p \mathbb{I}, & \text { in } B, \\
\operatorname{div} \boldsymbol{u}+\frac{p}{\lambda} & =0, & \text { in } B, \\
\boldsymbol{u} & =\boldsymbol{U}, & \text { on } \partial_{D} B, \\
\sigma \cdot \boldsymbol{n} & =\boldsymbol{T}, & \text { on } \partial_{N} B,
\end{aligned}
$$

where $\boldsymbol{f} \in\left[L^{2}(B)\right]^{d}, \boldsymbol{U} \in\left[H^{\frac{3}{2}}\left(\partial_{D} B\right)\right]^{d}, \boldsymbol{T} \in\left[H^{\frac{1}{2}}\left(\partial_{N} B\right)\right]^{d}, \nabla^{\text {sym }}$ is the symmetric gradient operator, I denotes the $d \times d$ identity tensor, and $\lambda$ and $\mu$ are Lamé constants such that $0<\mu \leq \lambda \leq \infty$. Subsets of the boundary, $\partial_{D} B$ and $\partial_{N} B$, are such that $\partial_{D} B \cap \partial_{N} B=\emptyset, \overline{\partial_{D} B \cup \partial_{N} B}=\partial B$, and $\left|\partial_{D} B\right|>0$. Vector $\boldsymbol{n}$ is the exterior unit normal to $B$.

The case $\lambda<\infty$ with $\lambda / \mu \gg 1$ corresponds to the nearly incompressible limit while $\lambda=\infty$ corresponds to perfect incompressibility ${ }^{3}$. Note here that the quantity $p$, the pressure parameter, coincides with the pressure only for the case $\lambda=\infty^{4}$. Nevertheless, we call $p$ the pressure for the sake of simplicity.

For the incompressible case $\lambda=\infty$ with $\partial_{N} B=\emptyset$, we require that $\boldsymbol{U}$ satisfy a compatibility condition

$$
\int_{\partial B} \boldsymbol{U} \cdot \boldsymbol{n} \mathrm{d} \Gamma=0
$$

In the case of $\lambda<\infty$, eliminating $p$ from the (2.1b) and (2.1c) yields

$$
\sigma=\lambda \operatorname{div} \boldsymbol{u} \mathbb{I}+2 \mu \nabla^{\mathrm{sym}} \boldsymbol{u}=\mathbb{C}: \nabla^{\mathrm{sym}} \boldsymbol{u} .
$$

Here $\mathbb{C}$ is a fourth-order tensor defined as

$$
\mathbb{C}=\left[\lambda \delta_{i j} \delta_{k l}+\mu\left(\delta_{i k} \delta_{j l}+\delta_{i l} \delta_{j k}\right)\right] \boldsymbol{e}_{i} \otimes \boldsymbol{e}_{j} \otimes \boldsymbol{e}_{k} \otimes \boldsymbol{e}_{l},
$$

where $\boldsymbol{e}_{i}, i=1, \ldots, d$, denotes the $i$ th Cartesian basis vector, and an index repeating twice in the same term implies summation from 1 through $d$.

We adopt standard notations for Hilbert spaces: $\|\cdot\|_{k, \Omega}$ denotes the $H^{k}(\Omega)$-norm while $|\cdot|_{k, \Omega}$ denotes the corresponding semi-norm. The symbol $|\cdot|$ without subscripts denote the Euclidean norm. We use the symbol $L_{0}^{2}(B)$ to denote the space

$$
L_{0}^{2}(B) \equiv\left\{q \in L^{2}(B): \int_{B} q \mathrm{~d} B=0\right\}
$$

\footnotetext{
${ }^{3}$ Note that in the case of plane stress, $\lambda$ in (2.1c) is interpreted as $\bar{\lambda} \equiv 2 \lambda \mu /(\lambda+2 \mu)$, where $\lambda$ and $\mu$ are quantities for the three-dimensional case. The limit $\bar{\lambda} \rightarrow \infty$ corresponds to the case of $\nu \rightarrow-1^{+}$, which is admissible but does not imply near incompressibility. Nevertheless, we will still call this limit as the incompressible limit for simplicity.

${ }^{4}$ The (hydrostatic) pressure $p_{H}$ is related to the Cartesian components of $\sigma$ as $p_{H}=-\left(\sigma_{11}+\sigma_{22}+\sigma_{33}\right) / 3$ for either $d=2$ or $d=3$, where for the case $d=2$, the 3 -direction is orthogonal to the plane that contains the domain $B$. In particular, for plane stress loading, $\sigma_{33}=0$; for plain strain, $\sigma_{33}=\nu\left(\sigma_{11}+\sigma_{22}\right)$, where $\nu=\lambda /[2(\lambda+\mu)]$. In all cases, when $\lambda$ is large, $p_{H}$ and $p$ are very close.
} 


\subsection{Regularity assumptions}

\subsubsection{For the primary variables}

The existence and uniqueness of the solution is a well known result (see, e.g., [10,21]). In the special case of $\partial_{N} B=\emptyset$, when $\lambda<\infty$, the solution $(\boldsymbol{u}, p)$ is unique in $\left[H^{1}(B)\right]^{d} \times L^{2}(B)$ while when $\lambda=\infty$, it is unique in $\left[H^{1}(B)\right]^{d} \times\left(L^{2}(B) / \mathbb{R}\right)$.

We assume, in the case of $\partial_{N} B=\emptyset$, that there exists $C>0$ independent of $\boldsymbol{f}, \boldsymbol{U}$, and $\lambda$ such that ${ }^{5}$

$$
\|\boldsymbol{u}\|_{m, B}+|p|_{m-1, B} \leq C\left(|\boldsymbol{f}|_{m-2, B}+\|\boldsymbol{U}\|_{m-\frac{1}{2}, \partial B}\right), \quad \text { for some } m \in \mathbb{N} \text { such that } m \geq 2
$$

The case of $d=m=2$ with $B$ convex and with some restrictive assumptions on $\boldsymbol{U}$ is proved in Brenner and Sung [9].

\subsubsection{For a construction needed in the analysis}

We assume that for every $\Theta \in L_{0}^{2}(B)$ there always exists $\boldsymbol{v} \in\left[H_{0}^{1}(B)\right]^{d}$ such that

$$
\begin{aligned}
\operatorname{div} \boldsymbol{v} & =\Theta, \\
\|\boldsymbol{v}\|_{1, B} & \leq C\|\Theta\|_{0, B},
\end{aligned}
$$

where $C>0$ is independent of $\Theta$. A constructive proof of this result based on the regularity of the Laplacian in two-dimensions was given in [8], Lemma 11.2.3.

\section{Methods}

We now introduce the family of DG mixed methods for near and perfect incompressibility. We then present the displacement DG method that results from a choice of parameters in the mixed method.

\subsection{Spatial discretization}

We construct a family of meshes $\left\{\mathscr{T}_{h}\right\}$, each consisting of open simplices (triangles for $d=2$ and tetrahedra for $d=3$ ) to discretize $B$ such that $\overline{\bigcup_{E \in \mathscr{T}_{h}} E}=B$. Moreover, for any $E_{1}, E_{2} \in \mathscr{T}_{h}, \partial E_{1} \cap \partial E_{2}$ can only be $\emptyset$, a common vertex, a common edge, or a common face. We let $\boldsymbol{n}_{E}: \partial E \rightarrow \mathbb{R}^{d}, E \in \mathscr{T}_{h}$, denote the exterior unit normal on $\partial E$. Additionally, on $\partial B$, we require that if a face $e \subset \partial B$, then either $e \subset \partial_{D} B$ or $e \subset \partial_{N} B$.

We assume that $\left\{\mathscr{T}_{h}\right\}$ is quasi-uniform, i.e., there exists a positive constant $C$ such that

$$
\rho_{E} \geq C h, \quad \forall E \in \mathscr{T}_{h}, \quad \forall \mathscr{T}_{h},
$$

where $h \equiv \max _{E \in \mathscr{T}_{h}} h_{E}$, and $h_{E}$ and $\rho_{E}$ denote the diameter and inradius of $E$, respectively.

We let $\mathscr{E}_{h}$ denote the set of all element faces, in which faces shared between neighboring elements appear only once, and

$$
\Gamma_{h} \equiv \bigcup_{e \in \mathscr{E}_{h}} e
$$

We then define

$$
\mathscr{E}_{h}^{D} \equiv\left\{e \in \mathscr{E}_{h}: e \subset \partial_{D} B\right\}, \quad \mathscr{E}_{h}^{N} \equiv\left\{e \in \mathscr{E}_{h}: e \subset \partial_{N} B\right\}, \quad \mathscr{E}_{h}^{I} \equiv \mathscr{E}_{h} \backslash\left(\mathscr{E}_{h} D \cup \mathscr{E}_{h}^{N}\right), \quad \Gamma_{h}^{I} \equiv \bigcup_{e \in \mathscr{E}_{h}^{I}} e
$$

\footnotetext{
${ }^{5}$ In the case of $\lambda<\infty, p$ is unique in $L^{2}(B)$, but (2.2) does not provide an estimate of the mean of $p$ over $B$. This quantity can be determined from the data of the problem, i.e.,

$$
\frac{1}{|B|} \int_{B} p \mathrm{~d} B=-\frac{\lambda}{|B|} \int_{\partial B} \boldsymbol{U} \cdot \boldsymbol{n} \mathrm{d} \Gamma
$$
}


For each face $e \in \mathscr{E}_{h}$, we arbitrarily label a + side and a - side, and set $\boldsymbol{n}_{e}$ to be the unit normal of $e$ pointing from the - side to the + side. For $e \in \mathscr{E}_{h} D \cup \mathscr{E}_{h}^{N}$, we set $\boldsymbol{n}_{e}$ to be the exterior normal $\boldsymbol{n}$ on $\partial B$. Now we extend the definition of $\boldsymbol{n}: \partial B \rightarrow \mathbb{R}^{d}$ to be $\boldsymbol{n}: \Gamma_{h} \rightarrow \mathbb{R}^{d}, \boldsymbol{n}=\boldsymbol{n}_{e}$ on each $e \in \mathscr{E}_{h}$ almost everywhere.

Let $v$ be a tensor field of any order that is sufficiently regular to possess traces on $e \in \mathscr{E}_{h} \cup \cup \mathscr{E}_{h}$ or on both sides of $e \in \mathscr{E}_{h}$. We let $\left.v\right|_{e^{ \pm}}$denote the trace of $v$ taken from the \pm side of $e \in \Gamma_{h}^{I}$. Then for $v$, we define the jump and average operators on $e$ as:

$$
\llbracket v \rrbracket \equiv\left\{\begin{array} { l l } 
{ v | _ { e ^ { - } } - v | _ { e ^ { + } } , } & { e \in \mathscr { E } _ { h } , } \\
{ v , } & { e \in \mathscr { E } _ { h } , } \\
{ 0 , } & { e \in \mathscr { E } _ { h } ^ { N } , }
\end{array} \quad \{ v \} \equiv \left\{\begin{array}{ll}
\frac{1}{2}\left(\left.v\right|_{e^{-}}+\left.v\right|_{e^{+}}\right), & e \in \mathscr{E}_{h}, \\
v, & e \in \mathscr{E}_{h} \cup \cup \mathscr{E}_{h} N
\end{array}\right.\right.
$$

Note that although both $\llbracket \cdot \rrbracket$ and $\boldsymbol{n}$ depend on the choice of the + and - sides for each $e$, their product does not.

\subsection{The approximation spaces}

To define the various approximation spaces, we first define

$$
W_{h}^{(l)} \equiv\left\{w_{h} \in L^{2}(B):\left.w_{h}\right|_{E} \in P_{l}(E), \forall E \in \mathscr{T}_{h}\right\},
$$

where $P_{l}(E)$ with integer $l \geq 0$ denotes the space of polynomials of total degree less than or equal to $l$. We seek an approximate displacement in $V_{h}$, where

$$
V_{h} \equiv\left[W_{h}^{(k)}\right]^{d},
$$

for some $k \in \mathbb{N}$. For the chosen $k$, we introduce the short-hand notation $W_{h} \equiv W_{h}^{(k)}$. Then we let

$$
\hat{V} \equiv\left[H^{1}(B)\right]^{d}+V_{h}
$$

which is the space that contains the error in displacement. We also have an independent approximation space for the pressure $Q_{h} \equiv W_{h}^{\left(k_{p}\right)}$, where $k_{p}=k-1$ or $k$.

\subsection{The DG derivative}

We define approximations to displacement gradients via the $D G$ derivative, i.e., $D_{D G}: \hat{V} \rightarrow\left[L^{2}(B)\right]^{d \times d}$, where

$$
D_{D G} \boldsymbol{u} \equiv \nabla_{h} \boldsymbol{u}+R_{\boldsymbol{U}}(\llbracket \boldsymbol{u} \rrbracket)
$$

where $\nabla_{h}$ denotes differentiation within $\bigcup_{E \in \mathscr{T}_{h}} E$, and the lifting operator $R_{U}:\left[L^{2}\left(\Gamma_{h}\right)\right]^{d} \rightarrow W_{h}^{d \times d}$ is defined to satisfy

$$
\int_{B} R_{\boldsymbol{U}}(\llbracket \boldsymbol{v} \rrbracket): \gamma_{h} \mathrm{~d} B=-\int_{\Gamma_{h}} \llbracket \boldsymbol{v} \rrbracket \cdot\left\{\gamma_{h}\right\} \cdot \boldsymbol{n} \mathrm{d} \Gamma+\int_{\partial_{D} B} \boldsymbol{U} \cdot \gamma_{h} \cdot n \mathrm{~d} \Gamma, \quad \forall \gamma_{h} \in W_{h}^{d \times d} .
$$

We note here that since $\nabla_{h} V_{h} \subset W_{h}^{d \times d}, D_{D G} V_{h} \subseteq W_{h}^{d \times d}$.

Next we introduce $\operatorname{div}_{D G} \boldsymbol{u}$ to denote the trace of $D_{D G} \boldsymbol{u}$, i.e.,

$$
\operatorname{div}_{D G} \boldsymbol{u} \equiv \operatorname{tr} D_{D G} \boldsymbol{u}=\operatorname{div}_{h} \boldsymbol{u}+\operatorname{tr} R_{U}(\llbracket \boldsymbol{u} \rrbracket) .
$$

Note that since $R_{U}$ is an affine operator, so are $D_{D G}$ and $\operatorname{div}_{D G}$. We let $R$ denote the homogeneous part of the affine operator $R_{U}$, i.e.,

$$
\int_{B} R(\llbracket \boldsymbol{v} \rrbracket): \gamma_{h} \mathrm{~d} B=-\int_{\Gamma_{h}} \llbracket \boldsymbol{v} \rrbracket \cdot\left\{\gamma_{h}\right\} \cdot \boldsymbol{n} \mathrm{d} \Gamma, \quad \forall \gamma_{h} \in W_{h}^{d \times d} .
$$


As a result, we have, for all $\boldsymbol{v} \in \hat{V}$,

$$
R_{\boldsymbol{U}}(\llbracket \boldsymbol{v} \rrbracket)=R(\llbracket \boldsymbol{v} \rrbracket)-R(\boldsymbol{U}),
$$

where whenever we write $R(\boldsymbol{U}), \boldsymbol{U}$ is understood as its extension from $\partial_{D} B$ to $\Gamma_{h}$ by zero.

An important property of the (homogeneous) lifting operator $R$ is given by [15], Lemma 1, which relates $\llbracket \cdot \rrbracket$ and $R(\llbracket \cdot \rrbracket)$ :

$$
\begin{aligned}
\|R(\llbracket \boldsymbol{v} \rrbracket)\|_{0, B} & \leq C h^{-\frac{1}{2}}\|\llbracket \boldsymbol{v} \rrbracket\|_{0, \Gamma_{h}}, \quad \forall \boldsymbol{v} \in \hat{V}, \\
\left\|\llbracket \boldsymbol{v}_{h} \rrbracket\right\|_{0, \Gamma_{h}} & \leq C h^{\frac{1}{2}}\left\|R\left(\llbracket \boldsymbol{v}_{h} \rrbracket\right)\right\|_{0, B}, \quad \forall \boldsymbol{v}_{h} \in V_{h} .
\end{aligned}
$$

These inequalities are a direct consequence of the mesh quasi-uniformity and the finite dimensions of $V_{h}$ and $W_{h}^{d \times d}$.

Another important property of $R$ is the following equality:

$$
\int_{B} z_{h} \operatorname{tr} R(\llbracket \boldsymbol{v} \rrbracket) \mathrm{d} B=-\int_{\Gamma_{h}} \llbracket \boldsymbol{v} \rrbracket \cdot \boldsymbol{n}\left\{z_{h}\right\} \mathrm{d} \Gamma, \quad \forall z_{h} \in W_{h},
$$

which is obtained by setting $\gamma_{h}=z_{h} \mathbb{I} \in W_{h}^{d \times d}$ in (3.2). This equation indicates that the trace of the lifting operator only depends on normal jumps across faces.

Next we define for all $\boldsymbol{v} \in \hat{V}$,

$$
\begin{aligned}
D_{D G}^{0} \boldsymbol{v} & \equiv \nabla_{h} \boldsymbol{v}+R(\llbracket \boldsymbol{v} \rrbracket) \\
\operatorname{div}_{D G}^{0} \boldsymbol{v} & \equiv \operatorname{tr} D_{D G}^{0} \boldsymbol{v}=\operatorname{div}_{h} \boldsymbol{v}+\operatorname{tr} R(\llbracket \boldsymbol{v} \rrbracket),
\end{aligned}
$$

where $\operatorname{div}_{h}$ denotes the divergence operator restricted to within each $E \in \mathscr{T}_{h}$. Linear operators $D_{D G}^{0}$ and $\operatorname{div}_{D G}^{0}$ are the linear parts of $D_{D G}$ and $\operatorname{div}_{D G}$, respectively, obtained by setting $\boldsymbol{U}=\mathbf{0}$. We then have

$$
\begin{aligned}
D_{D G} \boldsymbol{v} & =D_{D G}^{0} \boldsymbol{v}-R(\boldsymbol{U}) \\
\operatorname{div}_{D G} \boldsymbol{v} & =\operatorname{div}_{D G}^{0} \boldsymbol{v}-\operatorname{tr} R(\boldsymbol{U}) .
\end{aligned}
$$

Finally, by choosing $z_{h}=1$ in (3.5) and applying the divergence theorem to each element, we obtain

$$
\int_{B} \operatorname{div}_{D G}^{0} \boldsymbol{v} \mathrm{d} B=\int_{\partial_{N} B} \boldsymbol{v} \cdot \boldsymbol{n} \mathrm{d} \Gamma
$$

In particular, if $\partial_{N} B=\emptyset$,

$$
\int_{B} \operatorname{div}_{D G}^{0} \boldsymbol{v} \mathrm{d} B=0
$$

\subsection{The mixed method for near and perfect incompressibility}

Our family of mixed methods is formulated as: find $\left(\boldsymbol{u}_{h}, p_{h}\right) \in V_{h} \times Q_{h}$, such that

$$
\begin{aligned}
\bar{a}_{h}\left(\boldsymbol{u}_{h}, \boldsymbol{v}_{h}\right)+b_{h}\left(\boldsymbol{v}_{h}, p_{h}\right) & =\bar{F}_{h}\left(\boldsymbol{v}_{h}\right), \quad \forall \boldsymbol{v}_{h} \in V_{h}, \\
b_{h}\left(\boldsymbol{u}_{h}, q_{h}\right)-c\left(p_{h}, q_{h}\right) & =g_{h}\left(q_{h}\right), \quad \forall q_{h} \in Q_{h},
\end{aligned}
$$


where

$$
\begin{aligned}
\bar{a}_{h}\left(\boldsymbol{u}_{h}, \boldsymbol{v}_{h}\right) & \equiv \int_{B} 2 \mu\left[D_{D G}^{0 \mathrm{sym}} \boldsymbol{u}_{h}: D_{D G}^{0 \mathrm{sym}} \boldsymbol{v}_{h}+\beta R\left(\llbracket \boldsymbol{u}_{h} \rrbracket\right): R\left(\llbracket \boldsymbol{v}_{h} \rrbracket\right)\right] \mathrm{d} B, \\
b_{h}\left(\boldsymbol{v}_{h}, q_{h}\right) & \equiv-\int_{B} q_{h} \operatorname{div}_{D G}^{0} \boldsymbol{v}_{h} \mathrm{~d} B \\
c\left(p_{h}, q_{h}\right) & \equiv \int_{B} \frac{1}{\lambda} p_{h} q_{h} \mathrm{~d} B \\
\bar{F}_{h}\left(\boldsymbol{v}_{h}\right) & \equiv \int_{B} \boldsymbol{f} \cdot \boldsymbol{v}_{h} \mathrm{~d} B+\int_{\partial_{N} B} \boldsymbol{T} \cdot \boldsymbol{v}_{h} \mathrm{~d} B+\int_{B} 2 \mu R(\boldsymbol{U}):\left[D_{D G}^{0 \text { sym }} \boldsymbol{v}_{h}+\beta R\left(\llbracket \boldsymbol{v}_{h} \rrbracket\right)\right] \mathrm{d} B, \\
g_{h}\left(q_{h}\right) & \equiv-\int_{B} q_{h} \operatorname{tr} R(\boldsymbol{U}) \mathrm{d} B,
\end{aligned}
$$

where $D_{D G}^{\text {0sym }}$ denotes the symmetric part of $D_{D G}^{0}$, and $\beta>0$ is a non-dimensional stabilization parameter. In the case of $\lambda=\infty$ and $\partial_{N} B=\emptyset, p_{h}$, like $p$, is unique up to an additive constant, i.e., $p_{h} \in Q_{h} / \mathbb{R}$.

The stress approximation is computed as

$$
\sigma_{h}=-p_{h} \mathbb{I}+2 \mu D_{D G}^{\mathrm{sym}} \boldsymbol{u}_{h}
$$

where $D_{D G}^{\text {sym }} \boldsymbol{u}_{h}$ denotes the symmetric part of $D_{D G} \boldsymbol{u}_{h}$.

Remark 3.1. From (3.6b) and (3.5), an alternative expression of (3.10b) is given by

$$
b\left(\boldsymbol{v}_{h}, q_{h}\right)=-\int_{B} q_{h} \operatorname{div}_{h} \boldsymbol{v}_{h} \mathrm{~d} B+\int_{\Gamma_{h}} \llbracket \boldsymbol{v}_{h} \rrbracket \cdot \boldsymbol{n}\left\{q_{h}\right\} \mathrm{d} \Gamma .
$$

\subsection{The displacement method for near incompressibility}

In the case of $\lambda<\infty$, if we choose $k_{p}=k$, then (3.9b) is equivalent to

$$
p_{h}=-\lambda \operatorname{div}_{D G} \boldsymbol{u}_{h} .
$$

Substituting (3.12) into (3.9a) yields the following displacement method: Find $\boldsymbol{u}_{h} \in V_{h}$, such that

$$
a_{h}\left(\boldsymbol{u}_{h}, \boldsymbol{v}_{h}\right)=F_{h}\left(\boldsymbol{v}_{h}\right), \quad \forall \boldsymbol{v}_{h} \in V_{h},
$$

where for any $\boldsymbol{u}_{h}, \boldsymbol{v}_{h} \in \hat{V}$,

$$
\begin{aligned}
a_{h}\left(\boldsymbol{u}_{h}, \boldsymbol{v}_{h}\right) & \equiv \int_{B}\left[D_{D G}^{0} \boldsymbol{u}_{h}: \mathbb{C}: D_{D G}^{0} \boldsymbol{v}_{h}+2 \beta \mu R\left(\llbracket \boldsymbol{u}_{h} \rrbracket\right): R\left(\llbracket \boldsymbol{v}_{h} \rrbracket\right)\right] \mathrm{d} B \\
F_{h}\left(\boldsymbol{v}_{h}\right) & \equiv \int_{B} \boldsymbol{f} \cdot \boldsymbol{v}_{h} \mathrm{~d} B+\int_{\partial_{N} B} \boldsymbol{T} \cdot \boldsymbol{v}_{h} \mathrm{~d} B+\int_{B} R(\boldsymbol{U}):\left[\mathbb{C}: D_{D G}^{0} \boldsymbol{v}_{h}+2 \beta \mu R\left(\llbracket \boldsymbol{v}_{h} \rrbracket\right)\right] \mathrm{d} B .
\end{aligned}
$$

The stress approximation in (3.11) can be equivalently stated as

$$
\sigma_{h}=\mathbb{C}: D_{D G} \boldsymbol{u}_{h}
$$

This method coincides with the method proposed in [30], except for the stabilization term. The stabilization term as given here was later adopted in [37].

Remark 3.2. Since this displacement method is only a particular case of the mixed one, we only need to prove the optimal convergence and uniformity in $\lambda$ for the mixed method, and those for the displacement method will follow. Despite this equivalence, the two methods differ by the number of unknowns. As a result, although the mixed method with $k_{p}=k$ per se does not show much advantage over the case with $k_{p}=k-1$, the equivalent displacement method does enjoy the advantage of having a smaller stiffness matrix. 
Remark 3.3. The displacement method (3.13) can be obtained, alternatively, by a variational principle which reads: Find $\boldsymbol{u}_{h} \in V_{h}$ that minimizes the functional $I_{h}: \hat{V} \rightarrow \mathbb{R}$, where

$$
I_{h}[\boldsymbol{u}] \equiv \frac{1}{2} \int_{B}\left[D_{D G} \boldsymbol{u}: \mathbb{C}: D_{D G} \boldsymbol{u}+2 \beta \mu R_{\boldsymbol{U}}(\llbracket \boldsymbol{u} \rrbracket): R_{\boldsymbol{U}}(\llbracket \boldsymbol{u} \rrbracket)\right] \mathrm{d} B-\int_{B} \boldsymbol{f} \cdot \boldsymbol{u} \mathrm{d} B-\int_{\partial_{N} B} \boldsymbol{T} \cdot \boldsymbol{u} \mathrm{d} B
$$

and the stationarity of $I_{h}$ yields (3.13).

Remark 3.4. The Dirichlet boundary conditions here, as well as in the mixed method, are weakly imposed. Strong enforcement of such boundary conditions can lead to volumetric locking in the limit of $\lambda \rightarrow \infty$, which deteriorates the method. We will discuss such phenomenon in Section 5.3.

Remark 3.5. As we have seen, both methods are obtained by replacing in the weak form the classical derivative $\nabla$ with $D_{D G}$, and adding a stabilization term to the symmetric bilinear form $a(\cdot, \cdot)$ or $\bar{a}(\cdot, \cdot)$. A direct consequence is that the form of the corresponding inf-sup condition for the mixed method appears almost like its counterpart for conforming methods except that we have $\operatorname{div}_{D G}^{0}$ in place of div.

\section{Proof of optimal COnvergence And Robustness}

In this section, we will confine ourselves to pure Dirichlet problems, i.e., $\partial_{N} B=\emptyset$. We will show that, under the regularity assumptions set forth in Section 2.2, the two methods introduced in Section 3 achieve optimal convergence with the generic constant $C$ independent of $\lambda$ as well as of $h, \boldsymbol{f}$, and $\boldsymbol{U}$, indicating the absence of locking.

\subsection{Main results}

We will work with the mesh-dependent norm $\||\cdot|\|_{S}: \hat{V} \rightarrow \mathbb{R}$, which is defined as

$$
\|\| \boldsymbol{v}\|\|_{S}^{2} \equiv\left\|\nabla_{h}^{\mathrm{sym}} \boldsymbol{v}\right\|_{0, B}^{2}+\|R(\llbracket \boldsymbol{v} \rrbracket)\|_{0, B}^{2}, \quad \forall \boldsymbol{v} \in \hat{V},
$$

where $\nabla_{h}^{\mathrm{sym}} v$ denotes the symmetric part of $\nabla_{h} v$. We will prove that $\|\cdot \mid\| \|_{S}$ is a norm in Theorem 4.7.

Next we remark on the existence and uniqueness of the solution to the approximate problem (3.9) in the space $V_{h} \times Q_{h}$ equipped with the norm $\|\cdot\| \cdot\left\|_{S}+\right\| \cdot \|_{0, B}$. When $\lambda<\infty$, this result directly follows from Theorem 4.20 to be introduced later. When $\lambda=\infty$, the existence and uniqueness are a consequence of Theorems 4.20 and 4.6 (see, e.g., [11]).

The primary result of this work is thus the optimal convergence of the primary fields $(\boldsymbol{u}$ and $p)$ in their "natural" norms (Thm. 4.1) with a constant independent of $\lambda$. Based on this result, we have also obtained error estimates of the $L^{2}$-norm of the displacement over the domain (Thm. 4.4) as well as along the faces (Cor. 4.2), and the convergence of the stress (Cor. 4.3).

Theorem 4.1 (convergence of the primary fields). Let $\partial_{N} B=\emptyset$ and let (u,p) be the solution to (2.1). Let $\left(\boldsymbol{u}_{h}, p_{h}\right)$ be the solution to (3.9). If $\boldsymbol{u} \in\left[H^{m}(B)\right]^{d}$ and $p \in H^{m-1}(B)$ for some $m \in \mathbb{N}$ such that $2 \leq m \leq k+1$, then there exists $C>0$ independent of $\boldsymbol{f}, \boldsymbol{U}, h$, and $\lambda$ such that

$$
\|\left.\left|\boldsymbol{u}-\boldsymbol{u}_{h}\right|\right|_{S} \leq C h^{m-1}\left(|\boldsymbol{u}|_{m, B}+|p|_{m-1, B}\right) .
$$

Moreover for $\lambda<\infty$, there exists $C>0$ independent of $\boldsymbol{f}, \boldsymbol{U}, h$, and $\lambda$ such that

$$
\left\|p-p_{h}\right\|_{0, B} \leq C h^{m-1}\left(|\boldsymbol{u}|_{m, B}+|p|_{m-1, B}\right),
$$

while for $\lambda=\infty$, there exists $C>0$ independent of $\boldsymbol{f}, \boldsymbol{U}$, and $h$ such that

$$
\left\|p-p_{h}\right\|_{L^{2}(B) / \mathbb{R}} \leq C h^{m-1}\left(|\boldsymbol{u}|_{m, B}+|p|_{m-1, B}\right) .
$$


Corollary 4.2 (convergence of the boundary condition and the intra-element discontinuities). Let the assumptions in Theorem 4.1 hold, then there exists $C>0$ independent of $\boldsymbol{f}, \boldsymbol{U}, h$, and $\lambda$ such that

$$
\left\|\llbracket \boldsymbol{u}-\boldsymbol{u}_{h} \rrbracket\right\|_{0, \Gamma_{h}} \leq C h^{m-\frac{1}{2}}\left(|\boldsymbol{u}|_{m, B}+|p|_{m-1, B}\right) .
$$

In particular,

$$
\left\|\boldsymbol{U}-\boldsymbol{u}_{h}\right\|_{0, \partial B} \leq C h^{m-\frac{1}{2}}\left(|\boldsymbol{u}|_{m, B}+|p|_{m-1, B}\right) .
$$

Corollary 4.3 (convergence of stress). Let the assumptions in Theorem 4.1 hold. Let $\sigma$ be given by (2.1b) and $\sigma_{h}$ by (3.11). In particular, in the case of $\lambda=\infty$, we interpret $p$ in (2.1b) and $p_{h}$ in (3.11) as elements of $L_{0}^{2}(B)$. Then there exists $C>0$ independent of $\boldsymbol{f}, \boldsymbol{U}, h$, and $\lambda$ such that

$$
\left\|\sigma-\sigma_{h}\right\|_{0, B} \leq C h^{m-1}\left(|\boldsymbol{u}|_{m, B}+|p|_{m-1, B}\right) .
$$

Theorem 4.4 (convergence in $\left.\|\cdot\|_{0, B}\right)$. Let the assumptions in Theorem 4.1 hold, then there exists $C>0$ independent of $\boldsymbol{f}, \boldsymbol{U}, h$, and $\lambda$ such that

$$
\left\|\boldsymbol{u}-\boldsymbol{u}_{h}\right\|_{0, B} \leq C h^{m}\left(|\boldsymbol{u}|_{m, B}+|p|_{m-1, B}\right) .
$$

Remark 4.5. Because of the equivalence of the displacement method (3.13) and the mixed method (3.9) in the case of $\lambda<\infty$ and $k_{p}=k$, the theorems and corollaries that we just stated in this section also apply to the solution obtained from (3.13) with $p_{h}$ given by (3.12).

Finally, because it is central to the results above, we state the following theorem on the satisfaction of the inf-sup condition for these methods.

Theorem 4.6 (inf-sup condition). The following inf-sup condition holds:

$$
\inf _{0 \neq q_{h} \in Q_{h}} \sup _{\mathbf{0} \neq \boldsymbol{v}_{h} \in V_{h}} \frac{b_{h}\left(\boldsymbol{v}_{h}, q_{h}\right)}{\left\|q_{h}\right\|_{L^{2}(B) / \mathbb{R}}\left\|\mid \boldsymbol{v}_{h}\right\|_{S}} \geq C_{I S},
$$

where $C_{I S}>0$ is independent of $h$.

\subsection{Overview of the analysis}

The critical step for the analysis is the proof of an inf-sup condition, Theorem 4.6, which is a necessary condition for the problem (3.9) to be well posed. As a preliminary step, we introduce the BDM [12] interpolation operator and its properties in Section 4.3. The BDM space explicitly shows that there exists a $\operatorname{div}_{D G^{0}}^{0}$-free subspace of the space of displacement $V_{h}$ that can optimally approximate any divergence-free displacement field in $\left[H^{1}(B)\right]^{d}$. As we discuss in Section 4.4, this is why the proposed methods are free of the locking problem. The proof of the inf-sup conditions for both choices of $k_{p}$ is given in Section 4.5. Here the case of $k_{p}=k-1$ is more straightforward to prove while the case of $k_{p}=k$ is accomplished by proving the surjectivity of the operator $\operatorname{div}_{D G}^{0}$ onto the pressure space $Q_{h} \cap L_{0}^{2}(B)$. With the inf-sup condition, the rest of the analysis is somewhat standard, which involves the continuity and coercivity of the bilinear operators, and a bound on the consistency error arising from the lack of Galerkin orthogonality. We will prove these ingredients in Section 4.6 and the main results in Section 4.7.

Henceforth the symbol $C$ is used to denote a generic positive constant independent of $\boldsymbol{f}, \boldsymbol{U}$, $h$, and $\lambda$, whose value may differ at each occurrence. Additional independence of $C$ will be specified.

Before beginning the analysis of the next section, we first prove that $\||\cdot|\|_{S}$ is a norm. 
Theorem 4.7. The functional $\|\cdot\| \|_{S}$ defined in (4.1) is a norm on $\hat{V}$.

Proof. It is evident that $\|\mid \cdot\| \|_{S}$ is a seminorm on $\hat{V}$. It suffices to show that for $\boldsymbol{v} \in \hat{V},\|\| \boldsymbol{v}\|\|_{S}=0$ implies $\|\boldsymbol{v}\|_{0, B}=0$.

To this end, we first note that if $\||\boldsymbol{v}|||_{S}=0$, then $\nabla_{h}^{\text {sym }} \boldsymbol{v}=R(\llbracket \boldsymbol{v} \rrbracket)=\mathbf{0}$. Thus, for any $E \in \mathscr{T}_{h},\left.\boldsymbol{v}\right|_{E}$ must be an affine function. Hence, $\boldsymbol{v} \in\left[W_{h}^{(1)}\right]^{d} \subseteq V_{h}$.

From the mesh quasi-uniformity, a Poincaré's inequality [7], (1.8) and a Korn's inequality [6], (1.19) for piecewise continuous spaces, and (3.4),

$$
\begin{aligned}
\|\boldsymbol{v}\|_{0, B}^{2} & \leq C\left(\left\|\nabla_{h} \boldsymbol{v}\right\|_{0, B}^{2}+h^{-1}\|\llbracket \boldsymbol{v} \rrbracket\|_{0, \Gamma_{h}^{I}}^{2}+\|\boldsymbol{v}\|_{0, \partial B}^{2}\right) \leq C\left(\left\|\nabla_{h}^{\mathrm{sym}} \boldsymbol{v}\right\|_{0, B}^{2}+h^{-1}\|\llbracket \boldsymbol{v} \rrbracket\|_{0, \Gamma_{h}}^{2}\right) \\
& \leq C h^{-1}\|\llbracket \boldsymbol{v} \rrbracket\|_{0, \Gamma_{h}}^{2} \leq C\|R(\llbracket \boldsymbol{v} \rrbracket)\|_{0, B}=0 .
\end{aligned}
$$

\subsection{Interpolation error estimates}

The BDM interpolation operator was introduced in [12] and [13] for two- and three-dimensions, respectively. The BDM interpolation operator of order $k$ maps any $\boldsymbol{u} \in\left[H^{1}(B)\right]^{d}$ to $\boldsymbol{u}_{I} \in V_{h} \cap H($ div; $B)$. Important properties of $\boldsymbol{u}_{I}$ which we shall take advantage of include:

$$
\begin{aligned}
& \operatorname{div} \boldsymbol{u}_{I}=\Pi_{P_{k-1}(E)}^{\perp} \operatorname{div} \boldsymbol{u}, \quad \text { in each } E \in \mathscr{T}_{h}, \\
& \operatorname{div} \boldsymbol{u}_{I}=\Pi_{W_{h}^{(k-1)}}^{\perp} \operatorname{div} \boldsymbol{u}, \quad \text { almost everywhere, } \\
& \int_{e}\left(\boldsymbol{u}-\boldsymbol{u}_{I}\right) \cdot \boldsymbol{n} p_{k} \mathrm{~d} \Gamma=0, \quad \forall p_{k} \in P_{k}(e), \forall e \in \mathscr{E}_{h},
\end{aligned}
$$

where $\Pi_{H}^{\perp}$ for some Hilbert space $H$ over domain $D$ denotes the $L^{2}(D)$-orthogonal projection into $H$.

We next summarize some interpolation properties of the BDM element. Such results with $s=0$ and 1 are given in [11-13], Chapter III; here we offer a proof for a more general $s$. A direct consequence of such properties is the interpolation error estimate given by Theorem 4.10.

Lemma 4.8 (local error estimates). If $\boldsymbol{u} \in\left[H^{m}(E)\right]^{d}$ with $m \in \mathbb{N}, m \leq k+1$, and $\boldsymbol{u}_{I}$ is the BDM interpolant of order $k$ of $\boldsymbol{u}$, then there exists $C>0$ independent of $\boldsymbol{u}$ such that for all $E \in \mathscr{T}_{h}$,

$$
\begin{aligned}
\left|\boldsymbol{u}-\boldsymbol{u}_{I}\right|_{s, E} & \leq C h^{m-s}|\boldsymbol{u}|_{m, E}, \quad s \in \mathbb{N}_{0}, s \leq m, \\
\left|\operatorname{div}\left(\boldsymbol{u}-\boldsymbol{u}_{I}\right)\right|_{s, E} & \leq C h^{m-1-s}|\operatorname{div} \boldsymbol{u}|_{m-1, E}, \quad s \in \mathbb{N}_{0}, s \leq m-1, \\
\| \boldsymbol{u}-\left.\boldsymbol{u}_{I}\right|_{0, \partial E} & \leq C h^{m-\frac{1}{2}}|\boldsymbol{u}|_{m, E}
\end{aligned}
$$

where $\mathbb{N}_{0} \equiv \mathbb{N} \cup\{0\}$.

Proof. Inequality (4.6a) follows from standard interpolation theories (see [17], Thm. 3.1.4). Inequality (4.6b) holds because of (4.3). Inequality (4.6c) is a direct consequence of: (a) a trace inequality with a scaling argument [11], pages 111-112.

$$
\|w\|_{0, \partial E}^{2} \leq C\left(h^{-1}\|w\|_{0, E}^{2}+h|w|_{1, E}^{2}\right), \quad \forall E \in \mathscr{T}_{h}, w \in H^{1}(E),
$$

and (b) inequality (4.6a) with $s=0,1$.

Corollary 4.9. There exists $C>0$ such that for every $\boldsymbol{u} \in\left[H^{m}(B)\right]^{d}, m \in \mathbb{N}, m \leq k+1$, its BDM interpolant of order $k, \boldsymbol{u}_{I}$, satisfies

$$
\begin{aligned}
\left\|\nabla_{h}\left(\boldsymbol{u}-\boldsymbol{u}_{I}\right)\right\|_{0, B} & \leq C h^{m-1}|\boldsymbol{u}|_{m, B}, \\
\left\|\operatorname{div}\left(\boldsymbol{u}-\boldsymbol{u}_{I}\right)\right\|_{0, B} & \leq C h^{m-1}|\operatorname{div} \boldsymbol{u}|_{m-1, B}, \\
\left\|\llbracket \boldsymbol{u}-\boldsymbol{u}_{I} \rrbracket\right\|_{0, \Gamma_{h}} & \leq C h^{m-\frac{1}{2}}|\boldsymbol{u}|_{m, B} .
\end{aligned}
$$


Theorem 4.10 (interpolation error estimate). There exists $C>0$ independent of $h$ such that for every $\boldsymbol{u} \in$ $\left[H^{m}(B)\right]^{d}, m \in \mathbb{N}, m \leq k+1$, its BDM interpolant of order $k, \boldsymbol{u}_{I}$, satisfies

$$
\|\left.\left|\boldsymbol{u}-\boldsymbol{u}_{I}\right|\right|_{S} \leq C h^{m-1}|\boldsymbol{u}|_{m, B}
$$

Proof. We first sum the square of (4.6a) with $s=1$ over all $E \in \mathscr{T}_{h}$ to obtain

$$
\left\|\nabla_{h}^{\mathrm{sym}}\left(\boldsymbol{u}-\boldsymbol{u}_{I}\right)\right\|_{0, B}^{2} \leq \sum_{E \in \mathscr{T}_{h}}\left|\boldsymbol{u}-\boldsymbol{u}_{I}\right|_{1, E}^{2} \leq C h^{2 m-2}|\boldsymbol{u}|_{m, B}^{2} .
$$

We then deduce that (4.8) holds if

$$
\left\|R\left(\llbracket \boldsymbol{u}-\boldsymbol{u}_{I} \rrbracket\right)\right\|_{0, B} \leq C h^{m-1}|\boldsymbol{u}|_{m, B},
$$

which can be obtained by applying (3.3) and (4.6c):

$$
\left\|R\left(\llbracket \boldsymbol{u}-\boldsymbol{u}_{I} \rrbracket\right)\right\|_{0, B}^{2} \leq C h^{-1}\left\|\llbracket \boldsymbol{u}-\boldsymbol{u}_{I} \rrbracket\right\|_{0, \Gamma_{h}}^{2} \leq C h^{-1} \sum_{E \in \mathscr{T}_{h}}\left\|\boldsymbol{u}-\boldsymbol{u}_{I}\right\|_{0, \partial E}^{2} \leq C h^{2 m-2}|\boldsymbol{u}|_{m, B}^{2} .
$$

Finally, from standard approximation theories (see [17], Thm. 3.1.4), if $p \in H^{m}(B)$ for some $m \in \mathbb{N}, p_{I}=$ $\Pi_{Q_{h}}^{\perp} p$, then

$$
\left|p-p_{I}\right|_{s, B} \leq C h^{m-s}|p|_{m, B}, \quad s=0,1
$$

Note that for $k_{p}=k$, although the exponent of $h$ in (4.9) can be one order higher, the overall order of convergence remains the same as the case of $k_{p}=k-1$.

\subsection{The locking-free property of the displacement space $V_{h}$}

Next we explain why our displacement space $V_{h}$ equipped with operator $\operatorname{div}_{D G}$ as an approximation of the (classical) divergence is locking free.

Proposition 4.11. Let $\boldsymbol{u}_{I}$ be the BDM interpolant of order $k$ of $\boldsymbol{u} \in\left[H^{1}(B)\right]^{d}$. Then

$$
\operatorname{div}_{D G}^{0}\left(\boldsymbol{u}-\boldsymbol{u}_{I}\right)=\operatorname{div}\left(\boldsymbol{u}-\boldsymbol{u}_{I}\right), \quad \text { almost everywhere. }
$$

Proof. We first note that from (3.6b), an equivalent equality is given by

$$
\operatorname{tr} R\left(\llbracket \boldsymbol{u}-\boldsymbol{u}_{I} \rrbracket\right)=0, \quad \text { almost everywhere. }
$$

Since by definition $\operatorname{tr} R\left(\llbracket \boldsymbol{u}-\boldsymbol{u}_{I} \rrbracket\right) \in W_{h},(4.10)$ is equivalent to

$$
\int_{E} p_{k} \operatorname{tr} R\left(\llbracket \boldsymbol{u}-\boldsymbol{u}_{I} \rrbracket\right) \mathrm{d} B=0, \quad \forall E \in \mathscr{T}_{h}, p_{k} \in P_{k}(E),
$$

which can be obtained by setting $\gamma_{h}=\chi_{E} p_{k} \mathbb{I}$ in (3.2), where $\chi_{E}$ denotes the characteristic function of $E$.

Corollary 4.12. There exists $C>0$ such that for every $\boldsymbol{u} \in\left[H^{m}(B)\right]^{d}, m \in \mathbb{N}, m \leq k+1$, its BDM interpolant of order $k, \boldsymbol{u}_{I}$, satisfies

$$
\left\|\operatorname{div}_{D G}^{0}\left(\boldsymbol{u}-\boldsymbol{u}_{I}\right)\right\|_{0, B} \leq C h^{m-1}|\operatorname{div} \boldsymbol{u}|_{m-1, B} .
$$

Proof. Summing the square of (4.6b) over all $E \in \mathscr{T}_{h}$ yields this inequality.

Remark 4.13. The reason for a conforming low-order method to lock is that when $\lambda \rightarrow \infty$, the incompressibility constraint $\operatorname{div} \boldsymbol{u}=0$ is imposed more and more strongly, and the kernel of div in the conforming space either cannot approximate $\boldsymbol{u}$ or can only approximate $\boldsymbol{u}$ to a suboptimal order.

In our case, however, if the exact solution $\boldsymbol{u}$ is such that $\operatorname{div} \boldsymbol{u}=0$, we have $\operatorname{div}_{D G} \boldsymbol{u}=0$, and by Corollary 4.12 ,

$$
\operatorname{div}_{D G} \boldsymbol{u}_{I}=\operatorname{div}_{D G}^{0} \boldsymbol{u}_{I}-\operatorname{tr} R(\boldsymbol{U})=\operatorname{div}_{D G}^{0} \boldsymbol{u}-\operatorname{tr} R(\boldsymbol{U})=\operatorname{div}_{D G} \boldsymbol{u}=0 .
$$

In the mean time, by Theorem $4.10, \boldsymbol{u}_{I} \in V_{h}$ can still approximate $\boldsymbol{u}$ to the optimal order in the norm $\||\cdot|\| \|_{S}$. This is the essential reason for the method to be free from the locking problem in the incompressible limit. 


\subsection{Proof of the inf-sup condition}

We will prove Theorem 4.6 by showing the surjectivity of $\operatorname{div}_{D G}^{0}$ into $Q_{h} \cap L_{0}^{2}(B)$ for both choices of $k_{p}$, which is given by Theorem 4.14. The proof of Theorem 4.6 then follows.

Theorem 4.14. For every $\Theta_{h} \in W_{h} \cap L_{0}^{2}(B)$, there exists $\boldsymbol{v}_{h} \in V_{h}$ such that

$$
\begin{aligned}
\operatorname{div}_{D G}^{0} \boldsymbol{v}_{h} & =\Theta_{h}, \\
\left\|\mid \boldsymbol{v}_{h}\right\| \|_{S} & \leq C\left\|\Theta_{h}\right\|_{0, B},
\end{aligned}
$$

where $C>0$ is independent of $h$ and $\Theta_{h}$.

We will prove Theorem 4.14 by explicitly constructing $\boldsymbol{v}_{h}$ for any given $\Theta_{h}$. To this end, we will first show in Lemma 4.15 that the regularity result (2.3) and a property of the BDM interpolant imply the result for a more restrictive class of $\Theta_{h}$, i.e., in the space $W_{h}^{(k-1)} \cap L_{0}^{2}(B)$. This will be sufficient for the analysis for $k_{p}=k-1$. For $k_{p}=k$, we need to accommodate the projection of $\Theta_{h} \in W_{h} \cap L_{0}^{2}(B)$ in the $L^{2}(B)$-orthogonal complement of $W_{h}^{(k-1)}$. We will accomplish this by proving Lemma 4.16 .

Lemma 4.15. For every $\Theta_{I} \in W_{h}^{(k-1)} \cap L_{0}^{2}(B)$, there exists $\boldsymbol{v}_{I} \in V_{h}$ such that

$$
\begin{aligned}
\operatorname{div}_{D G}^{0} \boldsymbol{v}_{I} & =\Theta_{I}, \\
\|\| \boldsymbol{v}_{I} \|_{S} & \leq C\left\|\Theta_{I}\right\|_{0, B},
\end{aligned}
$$

where $C>0$ is independent of $h$ and $\Theta_{I}$.

Proof. Under the regularity assumption (2.3), we can find $\boldsymbol{v} \in\left[H_{0}^{1}(B)\right]^{d}$ to be such that

$$
\begin{aligned}
\operatorname{div} \boldsymbol{v} & =\Theta_{I}, \\
\|\boldsymbol{v}\|_{1, B} & \leq C\left\|\Theta_{I}\right\|_{0, B},
\end{aligned}
$$

where $C>0$ is independent of $\Theta_{I}$. Since $\boldsymbol{v}=\mathbf{0}$ on $\partial B$, we have $\operatorname{div}_{D G}^{0} \boldsymbol{v}=\operatorname{div} \boldsymbol{v}$, and \|\| $\boldsymbol{v}\left\|\left.\right|_{S}=\right\| \nabla^{\mathrm{sym}} \boldsymbol{v} \|$.

We then set $\boldsymbol{v}_{I} \in V_{h}$ to be the BDM interpolant of order $k$ of $\boldsymbol{v}$. From Proposition 4.11 and (4.4), we have

$$
\operatorname{div}_{D G}^{0} \boldsymbol{v}_{I}=\operatorname{div} \boldsymbol{v}_{I}=\Pi_{W_{h}^{(k-1)}}^{\perp} \operatorname{div} \boldsymbol{v}=\Pi_{W_{h}^{(k-1)}}^{\perp} \Theta_{I}=\Theta_{I}, \quad \text { almost everywhere. }
$$

It remains to show $(4.11 \mathrm{~b})$, which is given by $(4.12 \mathrm{~b})$ and Theorem 4.10 with $m=1$ :

$$
\left.\left\|\left|\boldsymbol{v}_{I}\right|\right\|\right|_{S} \leq\left.\|\boldsymbol{v}\|\right|_{S}+\left\|\boldsymbol{v}-\boldsymbol{v}_{I}\left|\left\|_{S} \leq\right\| \nabla^{\mathrm{sym}} \boldsymbol{v}\left\|_{0, B}+C|\boldsymbol{v}|_{1, B} \leq C\right\| \Theta_{I} \|_{0, B} .\right.\right.
$$

To proceed, we define the $L^{2}(B)$-orthogonal complement of $W_{h}^{(k-1)}$ :

$$
S_{h} \equiv\left\{s_{h} \in W_{h}: \int_{B} s_{h} p_{k-1} \mathrm{~d} B=0, \forall p_{k-1} \in W_{h}^{(k-1)}\right\} .
$$

It is clear that $S_{h} \subset L_{0}^{2}(B)$, and as a result, the following decomposition holds:

$$
W_{h} \cap L_{0}^{2}(B)=\left[W_{h}^{(k-1)} \cap L_{0}^{2}(B)\right] \oplus S_{h} .
$$

Later we will also need to use the following space on $\Gamma_{h}$ :

$$
\left\{S_{h}\right\} \equiv\left\{\left.\left\{s_{h}\right\}\right|_{\Gamma_{h}}: s_{h} \in S_{h}\right\} .
$$


Lemma 4.16. For every $\Theta_{I I} \in S_{h}$, there exists $\boldsymbol{v}_{I I} \in V_{h}$ such that

$$
\begin{aligned}
\operatorname{div}_{D G}^{0} \boldsymbol{v}_{I I} & =\Theta_{I I}, \\
\|\| \boldsymbol{v}_{I I} \mid \|_{S} & \leq C\left\|\Theta_{I I}\right\|_{0, B} .
\end{aligned}
$$

where $C>0$ is independent of $h$ and $\Theta_{I I}$.

The proof of Lemma 4.16 is constructive. To this end, we first prove Lemmas 4.17 and 4.18.

Lemma 4.17 (trace inequality for $S_{h}$ ). There exists $C>0$ independent of $h$ such that

$$
\left\|s_{h}\right\|_{0, B} \leq C h^{\frac{1}{2}}\left\|\left\{s_{h}\right\}\right\|_{0, \Gamma_{h}}, \quad \forall s_{h} \in S_{h} .
$$

Proof. We will prove (4.14) by showing that $\|\{\cdot\}\|_{0, \Gamma_{h}}$ is a norm on $S_{h}$, and (4.14) follows from the norm equivalence of $\|\{\cdot\}\|_{0, \Gamma_{h}}$ and $\|\cdot\|_{0, B}$ and a scaling argument.

It is evident that $\|\{\cdot\}\|_{0, \Gamma_{h}}$ is a seminorm on $S_{h}$; it remains to show that for any $s_{h} \in S_{h},\left\|\left\{s_{h}\right\}\right\|_{0, \Gamma_{h}}=0$ implies $s_{h}=0$.

To this end, we first show that for any $E \in \mathscr{T}_{h}$ and $e \in \mathscr{E}_{h}, e \subset \partial E,\left.s_{h}\right|_{e}=0$ implies $\left.s_{h}\right|_{E}=0$. Let the equation of the line $(d=2)$ or the plane $(d=3)$ that contains $e$ be $r(\boldsymbol{x})=0$, such that $\left.r\right|_{E}>0$. Then there exists $p_{k-1} \in P_{k-1}(E)$ such that $s_{h}=r p_{k-1}$. But from (4.13), $s_{h}=0$.

Now consider $e \subset \partial B$. On such $e,\left\{s_{h}\right\}=s_{h}$. Hence, $\left\{s_{h}\right\}=0$ implies $s_{h}=0$ in all $E$ such that $|\partial E \cap \partial B|>0$. Continuing the argument towards the interior of the domain, we have $s_{h}=0$ in $B$.

To proceed, we let

$$
J_{h} \equiv \prod_{e \in \mathscr{E}_{h}} P_{k}(e)
$$

Lemma 4.18 (inverse inequality). For every $j_{h} \in J_{h}$, there exists $\boldsymbol{w}_{h} \in V_{h}$ such that

$$
\begin{aligned}
\llbracket \boldsymbol{w}_{h} \rrbracket \cdot \boldsymbol{n} & =j_{h}, \\
\left\|\boldsymbol{w}_{h}\right\|_{S} & \leq C h^{-\frac{1}{2}}\left\|j_{h}\right\|_{0, \Gamma_{h}},
\end{aligned}
$$

where $C>0$ is independent of $h$ and $j_{h}$.

Remark 4.19. A similar result was obtained in [15], in which the authors had

$$
\left\|\boldsymbol{w}_{h}\right\|_{0, B} \leq C h^{\frac{1}{2}}\left\|j_{h}\right\|_{0, \Gamma_{h}}
$$

instead of (4.15b).

Proof. We construct $\boldsymbol{w}_{h}$ using the BDM element. For each $E \in \mathscr{T}_{h}$, we set $\left.\boldsymbol{w}_{h}\right|_{E} \in\left[P_{k}(E)\right]^{d}$ to be uniquely determined by $[12,13]$

$$
\left\{\begin{array}{l}
\boldsymbol{w}_{h} \cdot \boldsymbol{n}_{E}=c_{e} j_{h}, \quad \text { on } e \subset \partial E \\
\int_{E} \boldsymbol{w}_{h} \cdot \nabla p_{k-1} \mathrm{~d} B=0, \quad \forall p_{k-1} \in P_{k-1}(E), \\
\int_{E} \boldsymbol{w}_{h} \cdot \phi_{k} \mathrm{~d} B=0, \quad \forall \phi_{k} \in \Phi_{k}(E),
\end{array}\right.
$$

where $c_{e}$ equals 1 if $e \subset \partial B$ and $\frac{1}{2}$ otherwise, and

$$
\Phi_{k}(E) \equiv\left\{\phi_{k} \in\left[P_{k}(E)\right]^{d}: \operatorname{div} \phi_{k}=0,\left.\phi_{k} \cdot \boldsymbol{n}_{E}\right|_{\partial E}=0\right\} .
$$


It is clear that $\llbracket \boldsymbol{w}_{h} \rrbracket \cdot \boldsymbol{n}=j_{h}$ on $\Gamma_{h}$. Moreover, observing that $\left.\boldsymbol{w}_{h}\right|_{E}$ linearly depends on $\left.j_{h}\right|_{\partial E}$, together with a scaling argument, we conclude that there exists $C>0$ independent of $j_{h}$ and $E$ such that

$$
\left\|\nabla^{\mathrm{sym}} \boldsymbol{w}_{h}\right\|_{0, E}^{2}+h^{-1}\left\|\boldsymbol{w}_{h}\right\|_{0, \partial E}^{2} \leq C h^{-1}\left\|j_{h}\right\|_{0, \partial E}^{2} .
$$

Applying (4.1) and (3.3) and summing (4.16) over all $E \in \mathscr{T}_{h}$ gives

$$
\left\|\boldsymbol{w}_{h}\right\|\left\|_{S}^{2} \leq\right\| \nabla_{h}^{\text {sym }} \boldsymbol{w}_{h}\left\|_{0, B}^{2}+C h^{-1}\right\| \llbracket \boldsymbol{w}_{h} \rrbracket\left\|_{0, \Gamma_{h}}^{2} \leq C \sum_{E \in \mathscr{T}_{h}}\left(\left\|\nabla_{h}^{\text {sym }} \boldsymbol{w}_{h}\right\|_{0, B}^{2}+h^{-1}\left\|\boldsymbol{w}_{h}\right\|_{0, \partial E}^{2}\right) \leq C h^{-1}\right\| j_{h} \|_{0, \Gamma_{h}}^{2},
$$

where $C>0$ is independent of $j_{h}$.

Now we are ready to proceed to prove Lemma 4.16, based on which we will prove Theorems 4.14 and 4.6.

Proof of Lemma 4.16. First we remark that if we can construct $\boldsymbol{w}_{h} \in V_{h}$ such that

$$
\begin{aligned}
\Pi_{S_{h}}^{\perp} \operatorname{div}_{D G}^{0} \boldsymbol{w}_{h} & =\Theta_{I I}, \\
\left\|\boldsymbol{w}_{h} \mid\right\| \|_{S} & \leq C\left\|\Theta_{I I}\right\|_{0, B},
\end{aligned}
$$

then from Lemma 4.15, we will also be able to find $\boldsymbol{v}_{I}$ for $\Theta_{I}=\Theta_{I I}-\operatorname{div}_{D G}^{0} \boldsymbol{w}_{h} \in W_{h}^{(k-1)} \cap L_{0}^{2}(B)$ such that

$$
\begin{aligned}
\operatorname{div}_{D G}^{0} \boldsymbol{v}_{I} & =\Theta_{I I}-\operatorname{div}_{D G}^{0} \boldsymbol{w}_{h}, \\
\left\|\boldsymbol{v}_{I} \mid\right\|_{S} & \leq C\left\|\Theta_{I I}-\operatorname{div}_{D G}^{0} \boldsymbol{w}_{h}\right\|_{0, B} .
\end{aligned}
$$

Then the construction of $\boldsymbol{v}_{I I}$ is complete if we set $\boldsymbol{v}_{I I}=\boldsymbol{v}_{I}+\boldsymbol{w}_{h}$, since now we have

$$
\operatorname{div}_{D G}^{0} \boldsymbol{v}_{I I}=\Theta_{I I}-\operatorname{div}_{D G}^{0} \boldsymbol{w}_{h}+\operatorname{div}_{D G}^{0} \boldsymbol{w}_{h}=\Theta_{I I},
$$

and

$$
\begin{aligned}
\left\|\boldsymbol{v}_{I I}\right\| \|_{S} & \leq\left.\left\|\left|\boldsymbol{v}_{I}\right|\right\|\right|_{S}+\left.\left\|\left|\boldsymbol{w}_{h}\right|\right\|\right|_{S} \\
& \leq C\left(\left\|\Theta_{I I}-\operatorname{div}_{D G}^{0} \boldsymbol{w}_{h}\right\|_{0, B}+\left\|\Theta_{I I}\right\|_{0, B}\right) \\
& \leq C\left(\left\|\Theta_{I I}\right\|_{0, B}+\left\|\operatorname{div}_{D G}^{0} \boldsymbol{w}_{h}\right\|_{0, B}\right) \\
& \leq C\left(\left\|\Theta_{I I}\right\|_{0, B}+\left\|\boldsymbol{w}_{h}\right\|_{S}\right) \\
& \leq C\left\|\Theta_{I I}\right\|_{0, B} .
\end{aligned}
$$

Now we construct $\boldsymbol{w}_{h} \in V_{h}$ that satisfies (4.17). We first note that since $\operatorname{div}_{h} \boldsymbol{w}_{h} \in W_{h}^{(k-1)}$, we have $\Pi_{S_{h}}^{\perp} \operatorname{div}_{h} \boldsymbol{w}_{h}=\mathbf{0}$, and thus from (3.6b),

$$
\Pi_{S_{h}}^{\perp} \operatorname{div}_{D G}^{0} \boldsymbol{w}_{h}=\Pi_{S_{h}}^{\perp} \operatorname{tr} R\left(\llbracket \boldsymbol{w}_{h} \rrbracket\right) .
$$

Hence, (4.17a) is equivalent to

$$
\int_{\Gamma_{h}} \llbracket \boldsymbol{w}_{h} \rrbracket \cdot \boldsymbol{n}\left\{\gamma_{h}\right\} \mathrm{d} \Gamma=-\int_{B} \Theta_{I I} \gamma_{h} \mathrm{~d} B, \quad \forall \gamma_{h} \in S_{h} .
$$

We next claim that the construction of $\boldsymbol{w}_{h} \in V_{h}$ that satisfies both (4.18) and (4.17b) is equivalent to finding $j_{h} \in\left\{S_{h}\right\}$ such that

$$
\begin{gathered}
\int_{\Gamma_{h}} j_{h}\left\{\gamma_{h}\right\} \mathrm{d} \Gamma=-\int_{B} \Theta_{I I} \gamma_{h} \mathrm{~d} B, \quad \forall \gamma_{h} \in S_{h}, \\
\left\|j_{h}\right\|_{0, \Gamma_{h}} \leq C h^{\frac{1}{2}}\left\|\Theta_{I I}\right\|_{0, B} .
\end{gathered}
$$


This is because if such $j_{h}$ exists, then from Lemma $4.18, \boldsymbol{w}_{h}$ will satisfy

$$
\begin{aligned}
& \llbracket \boldsymbol{w}_{h} \rrbracket \cdot \boldsymbol{n}=j_{h}, \\
& \left\|\boldsymbol{w}_{h}\right\|_{S} \leq C h^{-\frac{1}{2}}\left\|j_{h}\right\|_{0, \Gamma_{h}} \leq C\left\|\Theta_{I I}\right\|_{0, B} .
\end{aligned}
$$

As a result, this $\boldsymbol{w}_{h}$ satisfies (4.18), and thus (4.17a).

Now we proceed to construct $j_{h} \in\left\{S_{h}\right\}$ that satisfies (4.19). To this end, we apply the Riesz representation theorem to the space $\left\{S_{h}\right\}$ equipped with the $L^{2}\left(\Gamma_{h}\right)$-inner product and the following linear functional in $\left\{S_{h}\right\}^{\prime}$ :

$$
\theta_{h}:\left\{\gamma_{h}\right\} \mapsto-\int_{B} \Theta_{I I} \gamma_{h} \mathrm{~d} B
$$

to conclude that there exists a unique $j_{h} \in\left\{S_{h}\right\}$ that satisfies (4.19a), and that

$$
\left\|j_{h}\right\|_{0, \Gamma_{h}}=\left\|\theta_{h}\right\|_{\left\{S_{h}\right\}^{\prime}}=\sup _{0 \neq \gamma_{h} \in S_{h}} \frac{-\int_{B} \Theta_{I I} \gamma_{h} \mathrm{~d} B}{\left\|\left\{\gamma_{h}\right\}\right\|_{0, \Gamma_{h}}} \leq \sup _{0 \neq \gamma_{h} \in S_{h}} \frac{\left\|\Theta_{I I}\right\|_{0, B}\left\|\gamma_{h}\right\|_{0, B}}{\left\|\left\{\gamma_{h}\right\}\right\|_{0, \Gamma_{h}}} \leq C h^{\frac{1}{2}}\left\|\Theta_{I I}\right\|_{0, B} .
$$

Proof of Theorem 4.14. We apply Lemma 4.15 to $\Pi_{W_{h}^{(k-1)}}^{\perp} \Theta_{h}$ and Lemma 4.17 to $\Pi_{S_{h}}^{\perp} \Theta_{h}$ to conclude that there exist $\boldsymbol{v}_{I}, \boldsymbol{v}_{I I} \in V_{h}$ such that

$$
\begin{aligned}
\operatorname{div}_{D G}^{0} \boldsymbol{v}_{I} & =\Pi_{W_{h}^{(k-1)}}^{\perp} \Theta_{h}, \\
\operatorname{div}_{D G}^{0} \boldsymbol{v}_{I I} & =\Pi_{S_{h}}^{\perp} \Theta_{h},
\end{aligned}
$$

and that

$$
\begin{aligned}
\left\|\boldsymbol{v}_{I} \mid\right\|_{S} & \leq C\left\|\Pi_{W_{h}^{(k-1)}}^{\perp} \Theta_{h}\right\|_{0, B}, \\
\left\|\boldsymbol{v}_{I I} \mid\right\|_{S} & \leq C\left\|\Pi_{S_{h}}^{\perp} \Theta_{h}\right\|_{0, B} .
\end{aligned}
$$

Now the construction of $\boldsymbol{v}_{h}$ is completed by setting $\boldsymbol{v}_{h}=\boldsymbol{v}_{I}+\boldsymbol{v}_{I I}$.

Proof of Theorem 4.6. It is clear that adding a constant to $q_{h} \in Q_{h}$ does not change the value of the fraction in (4.2). Hence, it is sufficient to prove that for all $q_{h} \in Q_{h} \cap L_{0}^{2}(B)$,

$$
\sup _{\mathbf{0} \neq \boldsymbol{v}_{h} \in V_{h}} \frac{b_{h}\left(\boldsymbol{v}_{h}, q_{h}\right)}{\left\|\boldsymbol{v}_{h}|\||_{S}\right.} \geq C_{I S}\left\|q_{h}\right\|_{0, B}
$$

where $C_{I S}>0$ is independent of $h$ and $q_{h}$.

To this end, we apply Theorem 4.14 to $q_{h} \in Q_{h} \cap L_{0}^{2}(B) \subseteq W_{h} \cap L_{0}^{2}(B)$ and conclude that there exists $\tilde{\boldsymbol{v}}_{h} \in V_{h}$ such that

$$
\begin{aligned}
\operatorname{div}_{D G}^{0} \tilde{\boldsymbol{v}}_{h} & =q_{h}, \\
\left\||| \tilde{\boldsymbol{v}}_{h}\right\| \|_{S} & \leq C\left\|q_{h}\right\|_{0, B},
\end{aligned}
$$

where $C$ is independent of $q_{h}$. Hence,

$$
\sup _{\mathbf{0} \neq \boldsymbol{v}_{h} \in V_{h}} \frac{b_{h}\left(\boldsymbol{v}_{h}, q_{h}\right)}{\left\|\boldsymbol{v}_{h} \mid\right\|_{S}} \geq \frac{b_{h}\left(\tilde{\boldsymbol{v}}_{h}, q_{h}\right)}{\left\|\tilde{\boldsymbol{v}}_{h}\right\|_{S}}=\frac{\left\|q_{h}\right\|_{0, B}^{2}}{\left\|\tilde{\boldsymbol{v}}_{h} \mid\right\|_{S}} \geq C\left\|q_{h}\right\|_{0, B}
$$




\subsection{Properties of the bilinear operators}

We continue now with the proof of the continuity and coercivity of the bilinear forms in Theorem 4.20, and a bound on the consistency error which arises from the lack of Galerkin orthogonality in Theorem 4.21. These results, together with the inf-sup condition, establish the existence of solutions of the discrete problem (3.9) $[10,21]$.

Theorem 4.20 (continuity and coercivity of the bilinear operators). The bilinear form $\bar{a}_{h}(\cdot, \cdot)$ satisfies the following continuity and coercivity properties for any $\beta>0$ :

$$
\begin{aligned}
\left|\bar{a}_{h}(\boldsymbol{u}, \boldsymbol{v})\right| & \leq C\left|\left\|\boldsymbol { u } \left|\left\|\left.\right|_{S} \mid\right\| \boldsymbol{v}\|\|_{S}, \quad \forall \boldsymbol{u}, \boldsymbol{v} \in \hat{V},\right.\right.\right. \\
\bar{a}_{h}\left(\boldsymbol{u}_{h}, \boldsymbol{u}_{h}\right) & \geq\left.\alpha\left|\| \boldsymbol{u}_{h}\right|\right|_{S} ^{2}, \quad \forall \boldsymbol{u}_{h} \in V_{h},
\end{aligned}
$$

where $\alpha>0$ depends on $\beta$ but is independent of $h$ and $\lambda$.

The bilinear form $b_{h}(\cdot, \cdot)$ satisfies the following continuity property:

$$
\left|b_{h}(\boldsymbol{v}, q)\right| \leq C\left|\|\boldsymbol{v} \mid\|\left\|_{S}\right\| q \|_{0, B}, \quad \forall \boldsymbol{v} \in \hat{V}, q \in L^{2}(B),\right.
$$

Proof. Here we only provide the proof of (4.21) since the rest are elementary. To prove (4.21), we note that for any $\epsilon>0$, we have Young's inequality

$$
\begin{aligned}
\bar{a}_{h}\left(\boldsymbol{u}_{h}, \boldsymbol{u}_{h}\right) & =\int_{B} 2 \mu\left[D_{D G}^{0 \mathrm{sym}} \boldsymbol{u}_{h}: D_{D G}^{0 \mathrm{sym}} \boldsymbol{u}_{h}+\beta R\left(\llbracket \boldsymbol{u}_{h} \rrbracket\right): R\left(\llbracket \boldsymbol{u}_{h} \rrbracket\right)\right] \mathrm{d} B \\
& \geq 2 \mu\left[(1-\epsilon)\left\|\nabla_{h}^{\mathrm{sym}} \boldsymbol{u}_{h}\right\|_{0, B}^{2}+\left(1+\beta-\frac{1}{\epsilon}\right)\left\|R\left(\llbracket \boldsymbol{u}_{h} \rrbracket\right)\right\|_{0, B}^{2}\right] .
\end{aligned}
$$

Choosing $\epsilon=(1+\beta / 2)^{-1}$ and setting $\alpha=2 \mu \beta /(2+\beta)$ yields the desired result.

Theorem 4.21 (consistency error estimates). Let $(\boldsymbol{u}, p)$ and $\left(\boldsymbol{u}_{h}, p_{h}\right)$ be the solutions to (2.1) and (3.9), respectively. Let $\boldsymbol{u} \in\left[H^{m}(B)\right]^{d}$ and $p \in H^{m-1}(B)$ for some $m \in \mathbb{N}$ such that $2 \leq m \leq k+1$, then

$$
\begin{aligned}
\left|\bar{a}_{h}(\boldsymbol{u}, \boldsymbol{v})+b_{h}(\boldsymbol{v}, p)-\bar{F}_{h}(\boldsymbol{v})\right| & \leq C h^{m-\frac{3}{2}}\|\llbracket \boldsymbol{v} \rrbracket\|_{0, \Gamma_{h}}\left(|\boldsymbol{u}|_{m, B}+|p|_{m-1, B}\right), \quad \forall \boldsymbol{v} \in \hat{V}, \\
b_{h}(\boldsymbol{u}, q)-c(p, q)-g_{h}(q) & =0, \quad \forall q \in L^{2}(B) .
\end{aligned}
$$

Proof. The proof of (4.24) is straightforward. In fact, since $\boldsymbol{u}$ is the exact solution,

$$
R_{\boldsymbol{U}}(\llbracket \boldsymbol{u} \rrbracket)=R(\llbracket \boldsymbol{u} \rrbracket)-R(\boldsymbol{U})=\mathbf{0} .
$$

Hence, from (3.1) and the continuity of $\boldsymbol{u}$,

$$
\operatorname{div}_{D G} \boldsymbol{u}=\operatorname{div} \boldsymbol{u}+R_{\boldsymbol{U}}(\boldsymbol{u})=\operatorname{div} \boldsymbol{u} .
$$

From (3.10b), (3.10c), (3.10e), (3.7b), (4.26), and (2.1c),

$$
b_{h}(\boldsymbol{u}, q)-c(p, q)-g_{h}(q)=-\int_{B} q\left(\operatorname{div}_{D G} \boldsymbol{u}+\frac{p}{\lambda}\right) \mathrm{d} B=-\int_{B} q\left(\operatorname{div} \boldsymbol{u}+\frac{p}{\lambda}\right) \mathrm{d} B=0 .
$$

To prove (4.23) for any $\boldsymbol{v} \in \hat{V}$, we invoke (4.25) to obtain

$$
\begin{aligned}
& \bar{a}_{h}(\boldsymbol{u}, \boldsymbol{v})+b_{h}(\boldsymbol{v}, p)-\bar{F}_{h}(\boldsymbol{v})=\int_{B}\left[\left(2 \mu \nabla^{\mathrm{sym}} \boldsymbol{u}-p \mathbb{I}\right): D_{D G}^{0 \mathrm{sym}} \boldsymbol{v}-\boldsymbol{f} \cdot \boldsymbol{v}\right] \mathrm{d} B \\
& =\int_{B}\left[\sigma:\left(\nabla_{h} \boldsymbol{v}+R(\llbracket \boldsymbol{v} \rrbracket)\right)-\boldsymbol{f} \cdot \boldsymbol{v}\right] \mathrm{d} B \\
& =\sum_{E \in \mathscr{T}_{h}}(\int_{E}-\underbrace{(\operatorname{div} \sigma+\boldsymbol{f})}_{=\mathbf{0}} \cdot \boldsymbol{v} \mathrm{d} B+\int_{\partial E} \boldsymbol{v} \cdot \sigma \cdot \boldsymbol{n}_{E} \mathrm{~d} \Gamma)+\int_{B} \sigma: R(\llbracket \boldsymbol{v} \rrbracket) \mathrm{d} B \\
& =\int_{\Gamma_{h}} \llbracket \boldsymbol{v} \rrbracket \cdot \sigma \cdot \boldsymbol{n} \mathrm{d} \Gamma+\int_{B} \sigma: R(\llbracket \boldsymbol{v} \rrbracket) \mathrm{d} B .
\end{aligned}
$$


We then define $\sigma_{I} \equiv 2 \mu \nabla_{h}^{\mathrm{sym}} \boldsymbol{u}_{I}-p_{I} \mathbb{I} \in W_{h}^{d \times d}$ where $\boldsymbol{u}_{I}$ is the BDM interpolant of order $k$ of $\boldsymbol{u}$ and $p_{I} \equiv \Pi_{Q_{h}}^{\perp} p$. From (3.2),

$$
\int_{\Gamma_{h}} \llbracket \boldsymbol{v} \rrbracket \cdot\left\{\sigma_{I}\right\} \cdot \boldsymbol{n} \mathrm{d} \Gamma+\int_{B} \sigma_{I}: R(\llbracket \boldsymbol{v} \rrbracket) \mathrm{d} B=0 .
$$

Subtracting (4.28) from (4.27) yields

$$
\bar{a}_{h}(\boldsymbol{u}, \boldsymbol{v})+b_{h}(\boldsymbol{v}, p)-\bar{F}_{h}(\boldsymbol{v})=\int_{\Gamma_{h}} \llbracket \boldsymbol{v} \rrbracket \cdot\left\{\sigma-\sigma_{I}\right\} \cdot \boldsymbol{n} \mathrm{d} \Gamma+\int_{B}\left(\sigma-\sigma_{I}\right): R(\llbracket \boldsymbol{v} \rrbracket) \mathrm{d} B .
$$

And hence, with (3.3) and a classical trace inequality with a scaling argument [11], pages 111-112,

$$
\begin{aligned}
\left|\bar{a}_{h}(\boldsymbol{u}, \boldsymbol{v})+b_{h}(\boldsymbol{v}, p)-\bar{F}_{h}(\boldsymbol{v})\right| & \leq\|\boldsymbol{v}\|_{0, \Gamma_{h}}\left\|\left\{\sigma-\sigma_{I}\right\}\right\|_{0, \Gamma_{h}}+\left\|\sigma-\sigma_{I}\right\|_{0, B}\|R(\llbracket \boldsymbol{v} \rrbracket)\|_{0, B} \\
& \leq C\|\boldsymbol{v}\|_{0, \Gamma_{h}}\left[\left\|\left\{\sigma-\sigma_{I}\right\}\right\|_{0, \Gamma_{h}}+h^{-\frac{1}{2}}\left\|\sigma-\sigma_{I}\right\|_{0, B}\right] .
\end{aligned}
$$

It remains to prove that

$$
\left\|\left\{\sigma-\sigma_{I}\right\}\right\|_{0, \Gamma_{h}}+h^{-\frac{1}{2}}\left\|\sigma-\sigma_{I}\right\|_{0, B} \leq C h^{m-\frac{3}{2}}\left(|\boldsymbol{u}|_{m, B}+|p|_{m-1, B}\right),
$$

which directly follows from a trace inequality with a scaling argument [11], page 111-112,

$$
\left\|\sigma-\sigma_{I}\right\|_{0, \partial E}^{2} \leq C\left(h\left|\sigma-\sigma_{I}\right|_{1, E}^{2}+h^{-1}\left\|\sigma-\sigma_{I}\right\|_{0, E}^{2}\right),
$$

where $C$ is independent of $E$, and interpolation error estimates (4.6a) and (4.9).

Remark 4.22. If we apply (4.23) to $\boldsymbol{v}_{h} \in V_{h}$, then from (3.4) and (4.1), we have

$$
\sup _{\mathbf{0} \neq \boldsymbol{v}_{h} \in V_{h}} \frac{\left|\bar{a}_{h}\left(\boldsymbol{u}, \boldsymbol{v}_{h}\right)+b_{h}\left(\boldsymbol{v}_{h}, p\right)-\bar{F}_{h}\left(\boldsymbol{v}_{h}\right)\right|}{|| \boldsymbol{v}_{h}||_{S}} \leq C h^{m-1}\left(|\boldsymbol{u}|_{m, B}+|p|_{m-1, B}\right),
$$

which implies that the consistency error optimally scales with $h$.

\subsection{Proof of the main results}

We are now ready to prove the main results.

Proof of Theorem 4.1. We first show that when $\lambda<\infty, p$ and $p_{h}$ have the same average over $B$, so that the subsequent analysis for $\lambda<\infty$ and $\lambda=\infty$ can be presented in a unified way. Let

$$
p_{0} \equiv \frac{1}{|B|} \int_{B} p \mathrm{~d} B, \quad p_{h 0} \equiv \frac{1}{|B|} \int_{B} p_{h} \mathrm{~d} B
$$

We then take $q_{h}=1$ in (3.9b) and applying (3.8), (3.5) and (2.1c) to obtain:

$$
-\frac{1}{\lambda} p_{h 0}|B|=-\int_{B} \operatorname{tr} R(\boldsymbol{U}) \mathrm{d} B=-\int_{\partial B} \boldsymbol{U} \cdot \boldsymbol{n} \mathrm{d} \Gamma=-\int_{B} \operatorname{div} \boldsymbol{u} \mathrm{d} B=-\frac{1}{\lambda} p_{0}|B| .
$$

Therefore,

$$
p_{h 0}=p_{0},
$$

and as a result, we only need to show for $\lambda \leq \infty$,

$$
||\left|\boldsymbol{u}-\boldsymbol{u}_{h}\right|||_{S}+\left\|p-p_{h}\right\|_{L^{2}(B) / \mathbb{R}} \leq C h^{m-1}\left(|\boldsymbol{u}|_{m, B}+|p|_{m-1, B}\right) .
$$


Let $\boldsymbol{u}_{I}$ be the BDM interpolant of order $k$ of $\boldsymbol{u}$ and $p_{I} \equiv \Pi_{Q_{h}}^{\perp} p$. Because of the interpolation error estimates (4.8) and (4.9), we only need to show

$$
||\left|\boldsymbol{u}_{h}-\boldsymbol{u}_{I}\right|||_{S}+\left\|p_{h}-p_{I}\right\|_{L^{2}(B) / \mathbb{R}} \leq C h^{m-1}\left(|\boldsymbol{u}|_{m, B}+|p|_{m-1, B}\right) .
$$

To this end, we invoke (3.9), (4.24), and the identity $c\left(p-p_{I}, q_{h}\right)=0$ to obtain

$$
\begin{aligned}
\bar{a}_{h}\left(\boldsymbol{u}_{h}-\boldsymbol{u}_{I}, \boldsymbol{v}_{h}\right)+b_{h}\left(\boldsymbol{v}_{h}, p_{h}-p_{I}\right) & =\tilde{F}_{h}\left(\boldsymbol{v}_{h}\right), \quad \forall \boldsymbol{v}_{h} \in V_{h}, \\
b_{h}\left(\boldsymbol{u}_{h}-\boldsymbol{u}_{I}, q_{h}\right)-c\left(p_{h}-p_{I}, q_{h}\right) & =\tilde{g}_{h}\left(q_{h}\right), \quad \forall q_{h} \in Q_{h},
\end{aligned}
$$

where

$$
\begin{aligned}
\tilde{F}_{h}\left(\boldsymbol{v}_{h}\right) & \equiv \bar{F}_{h}\left(\boldsymbol{v}_{h}\right)-\bar{a}_{h}\left(\boldsymbol{u}_{I}, \boldsymbol{v}_{h}\right)-b_{h}\left(\boldsymbol{v}_{h}, p_{I}\right), \\
\tilde{g}_{h}\left(q_{h}\right) & \equiv b_{h}\left(\boldsymbol{u}-\boldsymbol{u}_{I}, q_{h}\right) .
\end{aligned}
$$

We remark that from (4.4), if $k_{p}=k-1, \tilde{g}_{h} \equiv 0$.

The coercivity of $a_{h}(\cdot, \cdot),(4.21)$, the inf-sup condition (4.2), and the finite dimensions of $Q_{h}$ allow us to apply Theorem 1.2 in [11], Chapter II, to obtain

$$
\left\|\boldsymbol{u}_{h}-\boldsymbol{u}_{I}\right\|_{S}+\left\|p_{h}-p_{I}\right\|_{L^{2}(B) / \mathbb{R}} \leq K\left(\left\|\tilde{F}_{h}\right\|_{V_{h}^{\prime}}+\left\|\tilde{g}_{h}\right\|_{Q_{h}^{\prime}}\right),
$$

where $K$ is a nonlinear function of $\left\|a_{h}\right\|, 1 / \lambda, 1 / \alpha$, and $1 / C_{I S}$ that is bounded on bounded subsets of these values. From Theorems 4.6 and 4.20, $K$ is independent of $h$ and $\lambda$ and hence will be denoted by $C$.

It remains to prove

$$
\left\|\tilde{F}_{h}\right\|_{V_{h}^{\prime}},\left\|\tilde{g}_{h}\right\|_{Q_{h}^{\prime}} \leq C h^{m-1}\left(|\boldsymbol{u}|_{m, B}+|p|_{m-1, B}\right)
$$

For $\tilde{F}_{h}$, we apply (4.30), (4.20), (4.22), Theorem 4.10, and (4.9) to obtain

$$
\begin{aligned}
\left\|\tilde{F}_{h}\right\|_{V_{h}^{\prime}} & \left.\leq \sup _{\substack{0 \neq \boldsymbol{v}_{h} \in V_{h}\\
}} \frac{\left|\bar{F}_{h}\left(\boldsymbol{v}_{h}\right)-\bar{a}_{h}\left(\boldsymbol{u}, \boldsymbol{v}_{h}\right)-b_{h}\left(\boldsymbol{v}_{h}, p\right)\right|}{\left\|\boldsymbol{v}_{h} \mid\right\|_{S}}+\sup _{\substack{0 \neq \boldsymbol{v}_{h} \in V_{h}\\
}} \frac{\left|\bar{a}_{h}\left(\boldsymbol{u}-\boldsymbol{u}_{I}, \boldsymbol{v}_{h}\right)\right|+\left|b_{h}\left(\boldsymbol{v}_{h}, p-p_{I}\right)\right|}{\| h^{m-1}\left(|\boldsymbol{u}|_{m, B}+|p|_{m-1, B}\right)+C\left(\left|\| \boldsymbol{u}-\boldsymbol{u}_{I}\right|||_{S}\right.}+\left\|p-p_{I}\right\|_{0, B}\right) \\
& \leq C h^{m-1}\left(|\boldsymbol{u}|_{m, B}+|p|_{m-1, B}\right) .
\end{aligned}
$$

Similarly, for $\tilde{g}_{h}$, from (4.22) and Theorem 4.10,

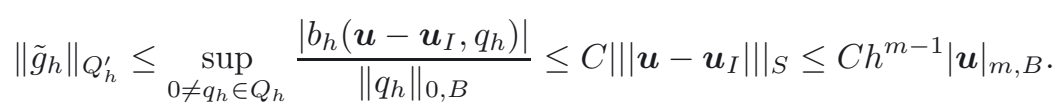

Proof of Corollary 4.2. We let $\boldsymbol{u}_{I}$ denote the BDM interpolant of order $k$ of $\boldsymbol{u}$ and apply (4.7c), (3.4), (4.31), and (4.1) to obtain

$$
\begin{aligned}
\left\|\llbracket \boldsymbol{u}-\boldsymbol{u}_{h} \rrbracket\right\|_{0, \Gamma_{h}} & \leq\left\|\llbracket \boldsymbol{u}-\boldsymbol{u}_{I} \rrbracket\right\|_{0, \Gamma_{h}}+\left\|\llbracket \boldsymbol{u}_{I}-\boldsymbol{u}_{h} \rrbracket\right\|_{0, \Gamma_{h}} \\
& \leq C h^{m-\frac{1}{2}}|\boldsymbol{u}|_{m, E}+C h^{\frac{1}{2}}\left\|R\left(\llbracket \boldsymbol{u}_{I}-\boldsymbol{u}_{h} \rrbracket\right)\right\|_{0, B} \\
& \leq C h^{m-\frac{1}{2}}|\boldsymbol{u}|_{m, E}+C h^{\frac{1}{2}}\left|\left\|\boldsymbol{u}_{I}-\boldsymbol{u}_{h} \mid\right\|_{S}\right. \\
& \leq C h^{m-\frac{1}{2}}\left(|\boldsymbol{u}|_{m, B}+|p|_{m-1, B}\right) .
\end{aligned}
$$

Proof of Corollary 4.3. From Theorem 4.1,

$$
\begin{aligned}
\left\|\boldsymbol{\sigma}-\boldsymbol{\sigma}_{h}\right\|_{0, B} & \leq 2 \mu\left\|\nabla^{\mathrm{sym}}\left(\boldsymbol{u}-\boldsymbol{u}_{h}\right)\right\|_{0, B}+\left\|p-p_{h}\right\|_{0, B} \\
& \leq C\left|\left\|\boldsymbol{u}-\boldsymbol{u}_{h} \mid\right\|_{S}+C h^{m-1}\left(|\boldsymbol{u}|_{m, B}+|p|_{m-1, B}\right)\right. \\
& \leq C h^{m-1}\left(|\boldsymbol{u}|_{m, B}+|p|_{m-1, B}\right) .
\end{aligned}
$$


Proof of Theorem 4.4. This theorem follows from an Aubin-Nitsche duality argument.

We set $(\boldsymbol{w}, q) \in\left[H_{0}^{1}(B)\right]^{d} \times L^{2}(B)$ to be such that

$$
\begin{aligned}
-\operatorname{div}\left(2 \mu \nabla^{\mathrm{sym}} \boldsymbol{w}-q \mathbb{I}\right) & =\boldsymbol{u}-\boldsymbol{u}_{h}, \quad \text { in } B, \\
\operatorname{div} \boldsymbol{w}+\frac{q}{\lambda} & =0, \quad \text { in } B .
\end{aligned}
$$

If $\lambda=\infty$, we further require $q \in L_{0}^{2}(B)$.

Then from (2.2), $\boldsymbol{w} \in\left[H^{2}(B)\right]^{d}, q \in H^{1}(B)$ and,

$$
\|\boldsymbol{w}\|_{2, B}+\|q\|_{1, B} \leq C\left\|\boldsymbol{u}-\boldsymbol{u}_{h}\right\|_{0, B} .
$$

Applying (4.23) to (4.36) with $m=2$ and $\boldsymbol{v}=\boldsymbol{u}-\boldsymbol{u}_{h} \in \hat{V}$ and invoking (4.37) and (4.35) yields

$$
\begin{aligned}
\left|\bar{a}_{h}\left(\boldsymbol{w}, \boldsymbol{u}-\boldsymbol{u}_{h}\right)+b_{h}\left(\boldsymbol{u}-\boldsymbol{u}_{h}, q\right)-\left\|\boldsymbol{u}-\boldsymbol{u}_{h}\right\|_{0, B}^{2}\right| & \leq C h^{\frac{1}{2}}\left\|\llbracket \boldsymbol{u}-\boldsymbol{u}_{h} \rrbracket\right\|_{0, \Gamma_{h}}\left(|\boldsymbol{w}|_{2, B}+|q|_{1, B}\right) \\
& \leq C h^{m}\left(|\boldsymbol{u}|_{m, B}+|p|_{m-1, B}\right)\left\|\boldsymbol{u}-\boldsymbol{u}_{h}\right\|_{0, B}
\end{aligned}
$$

or equivalently,

$$
\left\|\boldsymbol{u}-\boldsymbol{u}_{h}\right\|_{0, B}^{2} \leq\left|\bar{a}_{h}\left(\boldsymbol{w}, \boldsymbol{u}-\boldsymbol{u}_{h}\right)+b_{h}\left(\boldsymbol{u}-\boldsymbol{u}_{h}, q\right)\right|+C h^{m}\left(|\boldsymbol{u}|_{m, B}+|p|_{m-1, B}\right)\left\|\boldsymbol{u}-\boldsymbol{u}_{h}\right\|_{0, B} .
$$

It remains to show

$$
\left|\bar{a}_{h}\left(\boldsymbol{w}, \boldsymbol{u}-\boldsymbol{u}_{h}\right)+b_{h}\left(\boldsymbol{u}-\boldsymbol{u}_{h}, q\right)\right| \leq C h^{m}\left\|\boldsymbol{u}-\boldsymbol{u}_{h}\right\|_{0, B}\left(|\boldsymbol{u}|_{m, B}+|p|_{m-1, B}\right) .
$$

To this end, let $\boldsymbol{w}_{I}$ be the BDM interpolant of order $k$ of $\boldsymbol{w}$ and $q_{I}=\Pi_{Q_{h}}^{\perp} q$, then from (3.9a),

$$
\begin{aligned}
& \left|\bar{a}_{h}\left(\boldsymbol{w}, \boldsymbol{u}-\boldsymbol{u}_{h}\right)+b_{h}\left(\boldsymbol{u}-\boldsymbol{u}_{h}, q\right)\right| \\
& \leq \underbrace{\left|\bar{a}_{h}\left(\boldsymbol{u}, \boldsymbol{w}_{I}\right)+b_{h}\left(\boldsymbol{w}_{I}, p\right)-\bar{F}_{h}\left(\boldsymbol{w}_{I}\right)\right|}_{I}+\underbrace{\left|\bar{a}_{h}\left(\boldsymbol{w}-\boldsymbol{w}_{I}, \boldsymbol{u}-\boldsymbol{u}_{h}\right)\right|}_{I I}+\underbrace{\left|b_{h}\left(\boldsymbol{u}-\boldsymbol{u}_{h}, q\right)-b_{h}\left(\boldsymbol{w}_{I}, p-p_{h}\right)\right|}_{I I I} .
\end{aligned}
$$

It remains to bound the quantities $I, I I$, and $I I I$ as

$$
I, I I, I I I \leq C h^{m}\left(|\boldsymbol{w}|_{2, B}+|q|_{1, B}\right)\left(|\boldsymbol{u}|_{m, B}+|p|_{m-1, B}\right) .
$$

To bound $I I$, we apply (4.20) and Theorem 4.10 to obtain

$$
I I \leq\left. C||\left|\boldsymbol{w}-\boldsymbol{w}_{I}\right|||\right|_{S}|| \boldsymbol{u}-\left.\boldsymbol{u}_{h}||\right|_{S} \leq C h^{m}|\boldsymbol{w}|_{2, B}\left(|\boldsymbol{u}|_{m, B}+|p|_{m-1, B}\right) .
$$

To bound $I$, we apply the consistency error estimate (4.23) to write

$$
I \leq C h^{m-\frac{3}{2}}\left\|\llbracket \boldsymbol{w}_{I} \rrbracket\right\|_{0, \Gamma_{h}}\left(|\boldsymbol{u}|_{m, B}+|p|_{m-1, B}\right) .
$$

We then notice that since $\boldsymbol{w} \in\left[H_{0}^{1}(B)\right]^{d}, \llbracket \boldsymbol{w} \rrbracket=\mathbf{0}$; thus, from (4.7c),

$$
\left\|\llbracket \boldsymbol{w}_{I} \rrbracket\right\|_{0, \Gamma_{h}}=\left\|\llbracket \boldsymbol{w}-\boldsymbol{w}_{I} \rrbracket\right\|_{0, \Gamma_{h}} \leq C h^{\frac{3}{2}}|\boldsymbol{w}|_{2, B} .
$$

Combining (4.38) and (4.39) yields the desired bound for $I$.

Finally, to bound $I I I$, we first apply (4.24) to problems (3.9) and (4.36) to write, respectively,

$$
\begin{aligned}
b_{h}\left(\boldsymbol{u}-\boldsymbol{u}_{h}, q_{I}\right) & =c\left(p-p_{h}, q_{I}\right), \\
b_{h}\left(\boldsymbol{w}, p-p_{h}\right) & =c\left(q, p-p_{h}\right) .
\end{aligned}
$$




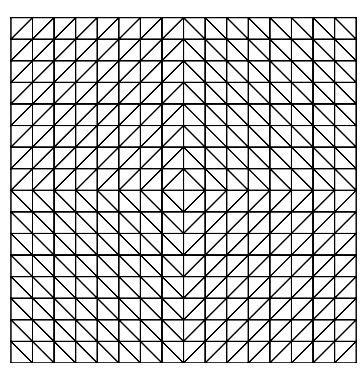

(a)

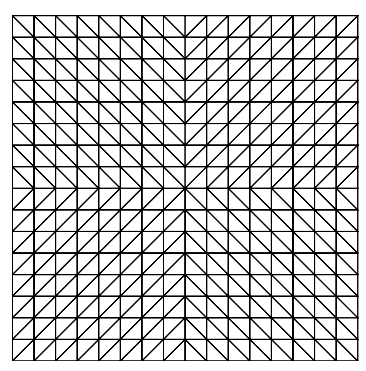

(b)

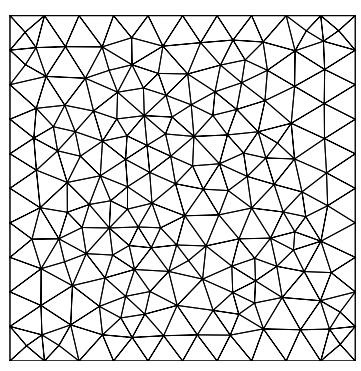

(c)

Figure 1. Two structured meshes [(a) and (b)] and one unstructured mesh [(c)] of the unit square $(0,1) \times(0,1)$ for the numerical examples.

We now can rewrite $I I I$ as

$$
\begin{aligned}
I I I & =\left|b_{h}\left(\boldsymbol{u}-\boldsymbol{u}_{h}, q_{I}\right)-b_{h}\left(\boldsymbol{w}_{I}, p-p_{h}\right)+b_{h}\left(\boldsymbol{u}-\boldsymbol{u}_{h}, q-q_{I}\right)\right| \\
& =\left|c\left(p-p_{h}, q_{I}\right)-b_{h}\left(\boldsymbol{w}_{I}, p-p_{h}\right)+b_{h}\left(\boldsymbol{u}-\boldsymbol{u}_{h}, q-q_{I}\right)\right| \\
& =\left|-c\left(p-p_{h}, q-q_{I}\right)+b_{h}\left(\boldsymbol{w}-\boldsymbol{w}_{I}, p-p_{h}\right)+b_{h}\left(\boldsymbol{u}-\boldsymbol{u}_{h}, q-q_{I}\right)\right| .
\end{aligned}
$$

The quantity $I I I$ can then be bounded by (4.22), Theorems 4.1, 4.10, and (4.9) as

$$
\begin{aligned}
I I I & \leq \frac{1}{\lambda}\left\|p-p_{h}\right\|_{0, B}\left\|q-q_{I}\right\|_{0, B}+C\left|\left\|\boldsymbol{w}-\boldsymbol{w}_{I}\left|\left\|\left.\right|_{S}\right\| p-p_{h}\left\|_{0, B}+C\left|\left\|\boldsymbol{u}-\boldsymbol{u}_{h} \mid\right\|\left\|_{S}\right\| q-q_{I} \|_{0, B}\right.\right.\right.\right.\right. \\
& \leq C h^{m}\left(|\boldsymbol{w}|_{2, B}+|q|_{1, B}\right)\left(|\boldsymbol{u}|_{m, B}+|p|_{m-1, B}\right) .
\end{aligned}
$$

\section{NumericAl EXAMPLES}

We will demonstrate in this section that our method optimally converges with a uniform error bound with respect to $\lambda$. We will also investigate the possible detrimental effect of strongly enforcing Dirichlet boundary conditions. In the following examples, we always set $k=1$ and $\beta=1$. The reader is reminded that any choice of $\beta>0$ yields a stable method.

\subsection{Optimal convergence with a uniform error bound}

We first demonstrate the robustness and optimal convergence of our methods. We consider the Dirichlet problem over the unit square $B=(0,1) \times(0,1)$ in two-dimensions corresponding to the exact solution

$$
\boldsymbol{u}=\mathrm{e}^{x}\left(\cos y \boldsymbol{e}_{1}-\sin y \boldsymbol{e}_{2}\right), \quad p=0 .
$$

It can be verified that $\operatorname{div} \boldsymbol{u}=0$, and that the stress field $\sigma$ is divergence-free and independent of $\lambda$.

We solved this problem with the proposed method with $\mathscr{T}_{h}$ defined by the three different meshes shown in Figure 1, and their recursive successive subdivisions. Such subdivisions were obtained by subdividing each triangle into four similar ones.

We first computed the errors in displacement and stress as functions of $h$ when we set $\lambda / \mu=10^{3}, \infty$ and plotted them in Figure 2. These plots demonstrate the optimal convergence rates attained by our methods $\left(h^{2}\right.$ for the displacement and $h$ for the stress).

To show that the error bounds are independent of $\lambda$, we plotted the normalized error in stress, $\| \sigma-$ $\sigma_{h}\left\|_{0, B} /\right\| \sigma \|_{0, B}$, as a function of Poisson's ratio $\nu \equiv \lambda /[2(\lambda+\mu)]$ in Figure 3 . We can see that with $k_{p}=0,1$ and all three meshes, $\sigma_{h}$ remains bounded as $\nu \rightarrow 0.5^{-}$, or $\lambda \rightarrow \infty$. 


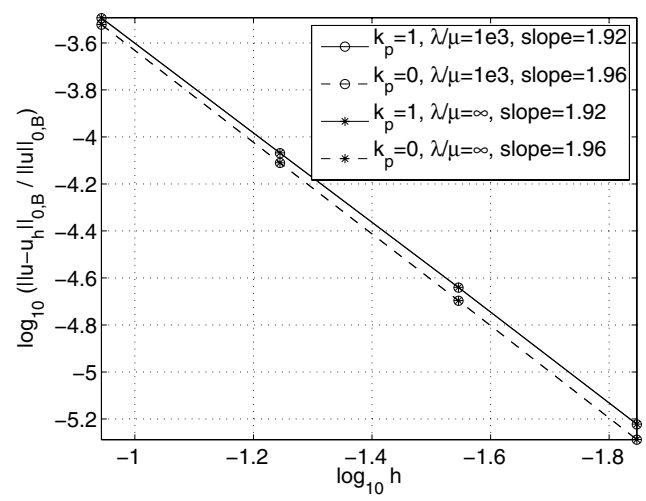

(a)

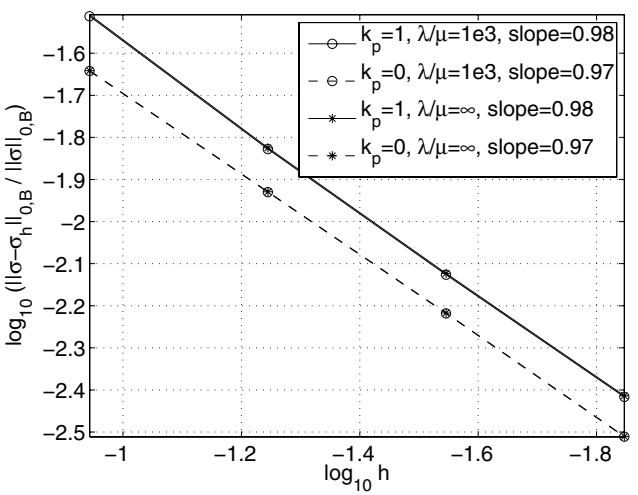

(b)

FiguRE 2. Demonstration of the optimal convergence rates of the methods. The mesh shown in Figure 1c and its recursive subdivisions are used to approximate the Dirichlet problem described in Section 5.1, with $\lambda / \mu=10^{3}, \infty$. The normalized $L^{2}$-norm of the error in (a) displacement and (b) stress are plotted as functions of $h$. As shown in the plots, the convergence rates are optimal ( $h^{2}$ for displacement and $h$ for stress).

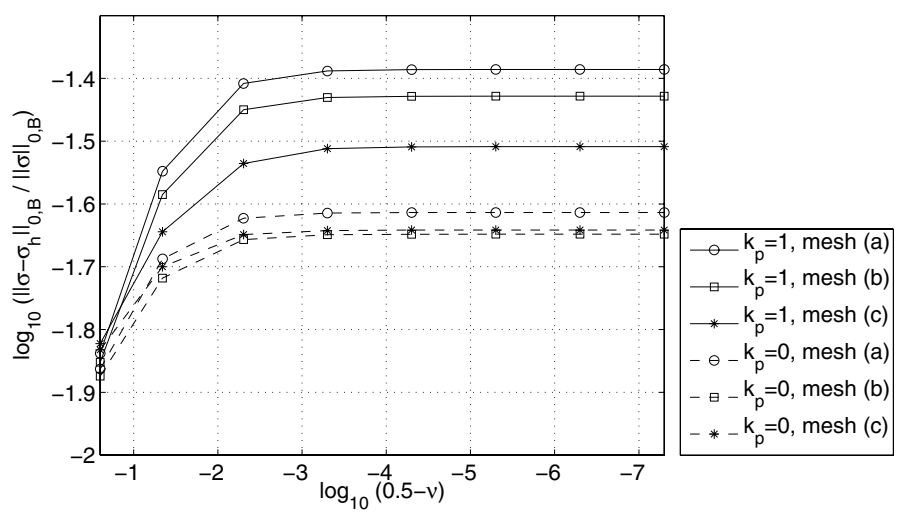

FiguRE 3. Demonstration of the uniform error bounds in the incompressible limit. The meshes shown in Figure 1 and their recursive subdivisions are used to approximate the Dirichlet problem described in Section 5.1, with $\lambda \rightarrow \infty$, or Poisson's ratio $\nu \equiv \lambda /[2(\lambda+\mu)] \rightarrow 0.5^{-}$. The normalized $L^{2}$-norm of the error in stress is plotted as a function of $\nu$. As shown in the plot, the stress remains bounded as $\nu \rightarrow 0.5^{-}$for all of these meshes and for both $k_{p}=0$ and $k_{p}=1$.

\subsection{The driven-cavity-flow-like problem}

We solve the following problem over the unit square $B=(0,1)^{2}$ :

$$
\begin{array}{rlrl}
\operatorname{div} \sigma & =\mathbf{0}, & & \text { in } B, \\
\sigma & =2 \mu \nabla^{\mathrm{sym}} \boldsymbol{u}-p \mathbb{I}, & & \text { in } B, \\
\operatorname{div} \boldsymbol{u}+\frac{p}{\lambda} & =0, & & \text { in } B, \\
\boldsymbol{u} & =\boldsymbol{e}_{1}, & \text { on }(0,1) \times\{1\}, \\
\boldsymbol{u} & =\mathbf{0}, & \text { on } \partial B \backslash[(0,1) \times\{1\}],
\end{array}
$$




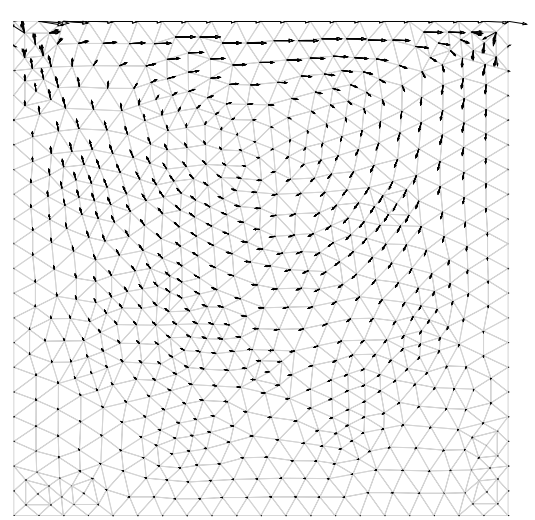

(a)

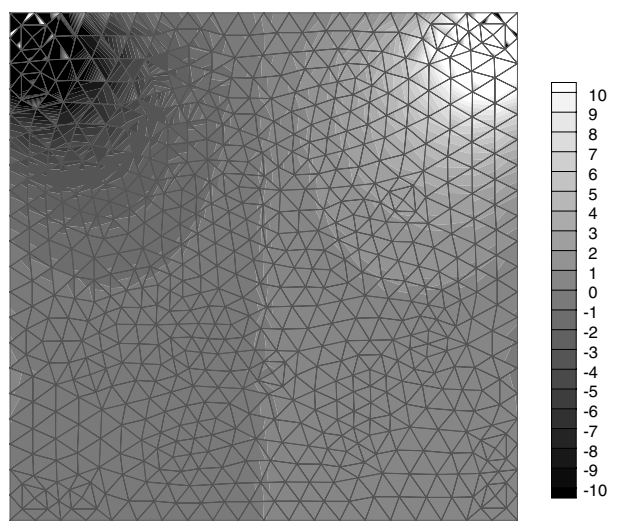

(b)

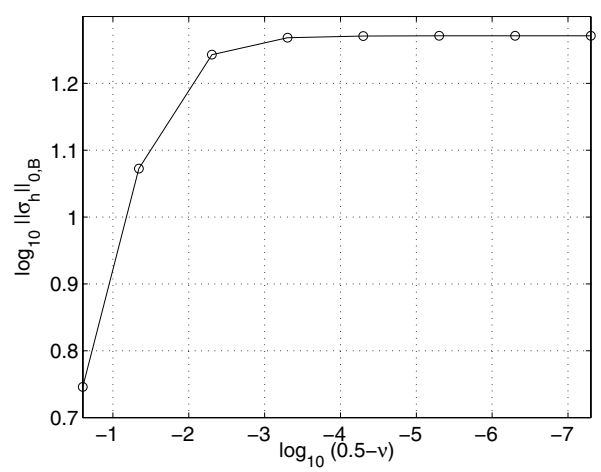

(c)

FIgURE 4. Solution of the driven-cavity-flow-like problem (5.1) which demonstrates the absence of locking of the displacement method: (a) vector plot of $\boldsymbol{u}_{h}$; (b) contour plot of $p_{h}$; (c) plot of $\left\|\sigma_{h}\right\|_{0, B}$ as a function of Poisson's ratio $\nu$. The absence of locking is evidenced in the displayed cavity in (a), the absence of spurious pressure mode in (b), and the boundedness of the pressure as $\nu \rightarrow 0.5^{-}$(c).

and with $\lambda / \mu$ up to $10^{7}$. If we let $\lambda \rightarrow \infty$, then (5.1) becomes the driven cavity flow problem. Here we will still use the terminology of elasticity.

The incompatibility in boundary conditions at two of the corners, $(0,1)$ and $(1,1)$, has two implications. First, $\boldsymbol{U}$ in this case is less regular than what would be required for optimal convergence rates. Nevertheless, we will demonstrate the absence of locking for this numerical example. Second, if we were to strongly enforce Dirichlet boundary conditions at the nodal points on the boundary, it would be necessary to specify the boundary conditions at these two corners, i.e., $\boldsymbol{e}_{1}$ versus $\mathbf{0}$, or some kind of average of them. Each choice would lead to a different solution. In our case, however, since we weakly imposed Dirichlet boundary conditions, we do not need to do so.

In Figure 4 we plot both the displacement and pressure obtained with our method with $k_{p}=1$ and $\lambda / \mu=$ $10^{7}$. We conclude that our method is locking-free because: (a) a single loop of vorticity is clearly visible in Figure 4a;(b) the stress field shown in Figure 4b displays no checker-board instability; and Figure 4c the stress remains bounded as $\lambda \rightarrow \infty$, as demonstrated in Figure 4c. 


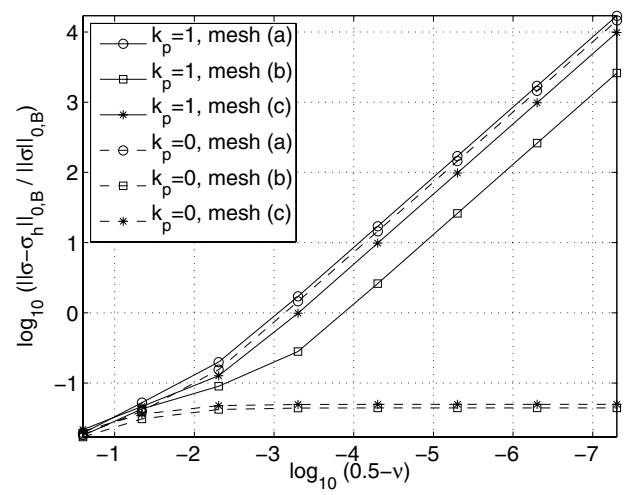

Figure 5. Demonstration of the possible locking effect caused by strong enforcement of Dirichlet boundary conditions. The normalized $L^{2}$-norm of the error in stress is plotted as a function of Poisson's ratio $\nu$, when the three meshes in Figure 1 are used to approximate the Dirichlet problem described in Section 5.1. Here we have altered the mixed method (3.9) by imposing the Dirichlet boundary conditions at all nodes on the boundary. It is clear that for all three meshes with $k_{p}=1$ and mesh (a) with $k_{p}=0$, the computed stress $\sigma_{h}$ goes unbounded as $\nu \rightarrow 0.5^{-}$, indicating the effect of locking. From the equivalence of the mixed method with $k=k_{p}$ and the displacement method, we conclude that the displacement method also locks if the Dirichlet boundary conditions are strongly imposed.

Note that as expected, the multiple displacement values at each node show a more pronounced difference near the two corners with incompatible boundary conditions.

\subsection{Consequences of strong enforcement of Dirichlet boundary conditions}

In this section we investigate the consequence of strong enforcement of Dirichlet boundary conditions. We will demonstrate that strong enforcement of Dirichlet boundary conditions may lead to locking in the incompressible limit.

To this end, we approximated the solution of the same problem as described in Section 5.1 with the same meshes shown in Figure 1 but with a slight modification in the way Dirichlet boundary conditions were imposed. In particular, we strongly enforced the boundary conditions at the nodes on the boundary. We then plotted the normalized error in stress, $\left\|\sigma-\sigma_{h}\right\|_{0, B} /\|\sigma\|_{0, B}$, as a function of $\nu$ in Figure 5 . For all three meshes with $k_{p}=1$ and mesh (a) with $k_{p}=0, \sigma_{h}$ becomes unbounded as $\nu \rightarrow 0.5^{-}$, which clearly displays the locking phenomenon. From Section 3.5, solving the same problem with these meshes and with the displacement method with strong enforcement of boundary conditions will also lead to locking.

In particular, note that from Figure 5 it follows that mesh (a) and its recursive subdivisions are more sensitive to strong imposition of boundary conditions than the other two, since locking appears with both values of $k_{p}$. The cause for this observation is that these meshes have four elements whose nodes are all located on $\partial B$, in contrast with the family of meshes (b) and (c). When imposing the boundary conditions strongly, this implies that the displacement field inside these elements is defined exclusively by the interpolated values, and may not be $\operatorname{div}_{D G}^{0}$-free, which leads to the observed locking behavior. For this reason, such elements have been explicitly avoided in the analysis of the Taylor-Hood element in [5], Proposition 1, for example.

We also note that in $[23,24,40]$, the Dirichlet boundary conditions were weakly imposed; while in [31], they were strongly imposed. 


\section{REFERENCES}

[1] D.N. Arnold, F. Brezzi and M. Fortin, A stable finite element for the Stokes equations. Calcolo 21 (1984) 337-344.

[2] D.N. Arnold, F. Brezzi, B. Cockburn and L.D. Marini, Unified analysis of discontinuous Galerkin methods for elliptic problems. SIAM J. Numer. Anal. 39 (2002) 1749-1779.

[3] F. Bassi and S. Rebay, A High-order accurate discontinuous finite element method for the numerical solution of the compressible Navier-Stokes equations. J. Comput. Phys. 131 (1997) 267-279.

[4] R. Becker, E. Burman and P. Hansbo, A Nitsche extended finite element method for incompressible elasticity with discontinuous modulus of elasticity. Comput. Methods Appl. Mech. Engrg. 198 (2009) 3352-3360.

[5] M. Bercovier and O.A. Pironneau, Error estimates for finite element method solution of the Stokes problem in the primitive variables. Numer. Math. 33 (1977) 211-224.

[6] S.C. Brenner, Korn's inequalities for piecewise $H^{1}$ vector fields. Math. Comp. 73 (2003) 1067-1087.

[7] S.C. Brenner, Poincaré-Friedrichs inequalities for piecewise $H^{1}$ functions. SIAM J. Numer. Anal. 41 (2003) $306-324$.

[8] S.C. Brenner and L.R. Scott, The mathematical theory of finite element methods, 3th edition, Springer (2008).

[9] S.C. Brenner and L.-Y. Sung, Linear finite element methods for planar linear elasticity. Math. Comp. 59 (1992) $321-338$.

[10] F. Brezzi, On the existence, uniqueness and approximation of saddle point problems arising from Lagrangian multipliers. RAIRO Anal. Numér. 8 (1974) 129-151.

[11] F. Brezzi and M. Fortin, Mixed and hybrid finite element methods. Springer Series in Computational Mathematics, SpringerVerlag, New York (1991).

[12] F. Brezzi, J. Douglas Jr., and L.D. Marini, Two families of mixed finite elements for second order elliptic problems. Numer. Math. 47 (1985) 217-235.

[13] F. Brezzi, J. Douglas Jr., R. Durán and M. Fortin, Mixed finite elements for second order elliptic problems in three variables. Numer. Math. 51 (1987) 237-250.

[14] F. Brezzi, G. Manzini, D. Marini, P. Pietra and A. Russo, Discontinuous galerkin approximations for elliptic problems. Numer. Methods Partial Differential Equations (2000) 365-378.

[15] F. Brezzi, T.J.R. Hughes, L.D. Marini and A. Masud, Mixed discontinuous Galerkin methods for Darcy flow. J. Sci. Comput. 22, 23 (2005) 119-145.

[16] J. Carrero, B. Cockburn and D. Schötzau, Hybridized globally divergence-free LDG methods. Part I: the Stokes problem. Math. Comp. 75 (2005) 533-563.

[17] P.G. Ciarlet, The finite element method for elliptic problems. North-Holland, Amsterdam (1978).

[18] B. Cockburn, G. Kanschat, D. Schötzau and C. Schwab, Local discontinuous Galerkin methods for the Stokes system. SIAM J. Numer. Anal. 40 (2002) 319-343.

[19] B. Cockburn, D. Schötzau and J. Wang, Discontinuous Galerkin methods for incompressible elastic materials. Comput. Methods Appl. Mech. Engrg. 195 (2006) 3184-3204.

[20] M. Crouzeix and P.-A. Raviart, Conforming and nonconforming finite element methods for solving the stationary Stokes equations. RAIRO Sér. Rouge 7 (1973) 33-75.

[21] M. Fortin, An analysis of the convergence of mixed finite element methods. RAIRO Anal. Numér. 11 (1977) $341-354$.

[22] V. Girault, B. Rivière and M.F. Wheeler, A discontinuous Galerkin method with nonoverlapping domain decomposition for the Stokes and Navier-Stokes problems. Math. Comp. 74 (2005) 53-84.

[23] P. Hansbo and M.G. Larson, Discontinuous Galerkin methods for incompressible and nearly incompressible elasticity by Nitsche's method. Comput. Methods Appl. Mech. Engrg. 191 (2002) 1895-1908.

[24] P. Hansbo and M.G. Larson, Discontinuous Galerkin and the Crouzeix-Raviart element: Application to elasticity. ESAIM: M2AN 37 (2003) 63-72.

[25] P. Hansbo and M.G. Larson, Piecewise divergence-free discontinuous Galerkin methods for Stokes flow. Comm. Num. Methods Engrg. 24 (2008) 355-366.

[26] F. Hecht, Construction d'une base de fonctions $P_{1}$ non conforme à divergence nulle dans $R^{3}$. RAIRO Anal. Numér. 15 (1981) 119-150.

[27] P. Hood and C. Taylor, Numerical solution of the Navier-Stokes equations using the finite element technique. Comput. Fluids 1 (1973) 1-28.

[28] P. Hood and C. Taylor, Navier-Stokes equations using mixed interpolation. Finite Element Methods in Flow Problems, edited by J.T. Oden. UAH Press, Huntsville, Alabama (1974).

[29] R. Kouhia and R. Stenberg, A linear nonconforming finite element method for nearly incompressible elasticity and Stokes flow. Comput. Methods Appl. Mech. Engrg. 124 (1995) 195-212.

[30] A. Lew, P. Neff, D. Sulsky and M. Ortiz, Optimal BV estimates for a discontinuous Galerkin method for linear elasticity. Appl. Math. Res. express 3 (2004) 73-106.

[31] N.C. Nguyen, J. Peraire and B. Cockburn, A hybridizable discontinuous Galerkin method for Stokes flow. Comput. Methods Appl. Mech. Engrg. 199 (2010) 582-597.

[32] B. Rivière and V. Girault, Discontinuous finite element methods for incompressible flows on subdomains with non-matching interfaces. Comput. Methods Appl. Mech. Engrg. 195 (2006) 3274-3292.

[33] D. Schötzau, C. Schwab and A. Toselli, Mixed hp-DGFEM for incompressible flows. SIAM J. Numer. Anal. 40 (2003) 21712194. 
[34] L.R. Scott and M. Vogelius, Norm estimates for a maximal right inverse of the divergence operator in spaces of piecewise polynomials. RAIRO Modélisation Mathématique et Analyse Numérique 19 (1985) 111-143.

[35] S.-C. Soon, B. Cockburn and H.K. Stolarski, A hybridizable discontinuous Galerkin method for linear elasticity. Int. J. Numer. Methods Engrg. 80 (2009), 1058-1092.

[36] A. Ten Eyck and A. Lew, Discontinuous Galerkin methods for non-linear elasticity. Int. J. Numer. Methods Engrg. 67 (2006) 1204-1243.

[37] A. Ten Eyck, F. Celiker and A. Lew, Adaptive stabilization of discontinuous Galerkin methods for nonlinear elasticity: Analytical estimates. Comput. Methods Appl. Mech. Engrg. 197 (2008) 2989-3000.

[38] F. Thomasset, Implementation of finite element methods for Navier-Stokes equations. Springer-Verlag, New York (1981).

[39] J.P. Whiteley, Discontinuous Galerkin finite element methods for incompressible non-linear elasticity, Comput. Methods Appl. Mech. Engrg. 198 (2009) 3464-3478.

[40] T.P. Wihler, Locking-free DGFEM for elasticity problems in polygons. IMA J. Numer. Anal. 24 (2004) 45-75. 